\title{
Topological states between inversion symmetric atomic insulators
}

\author{
Ana Silva ${ }^{1}$, Jasper van Wezel ${ }^{2 *}$
}

1 Department of Physics of Complex Systems, Weizmann Institute of Science, Rehovot 76100 , Israel

2 Institute for Theoretical Physics, Institute of Physics, University of Amsterdam, 1090

GL Amsterdam, The Netherlands

*vanwezel@uva.nl

January 7, 2021

\begin{abstract}
One of the hallmarks of topological insulators is the correspondence between the value of its bulk topological invariant and the number of topologically protected edge modes observed in a finite-sized sample. This bulk-boundary correspondence has been well-tested for strong topological invariants, and forms the basis for all proposed technological applications of topology. Here, we report that a group of weak topological invariants, which depend only by the symmetries of the atomic lattice, also induces a particular type of bulk-boundary correspondence. It predicts the presence or absence of states localised at the interface between two inversion-symmetric band insulators with trivial values for their strong invariants, based on the space group representation of the bands on either side of the junction. We show that this corresponds with symmetry-based classifications of topological materials. The interface modes are protected by the combination of band topology and symmetry of the interface, and may be used for topological transport and signal manipulation in heterojunction-based devices.
\end{abstract}

\section{Contents}

\begin{tabular}{lll}
\hline 1 & Introduction & 2
\end{tabular}

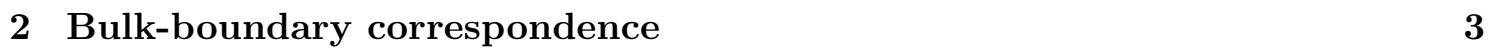

\begin{tabular}{|lll}
\hline 3 & Interface states & 4
\end{tabular}

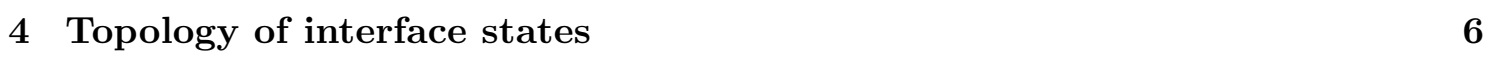

\begin{tabular}{llr}
\hline 5 & Discussion & 8
\end{tabular}

A Symmetry-imposed zeroes of Bloch wave functions and their deriva-

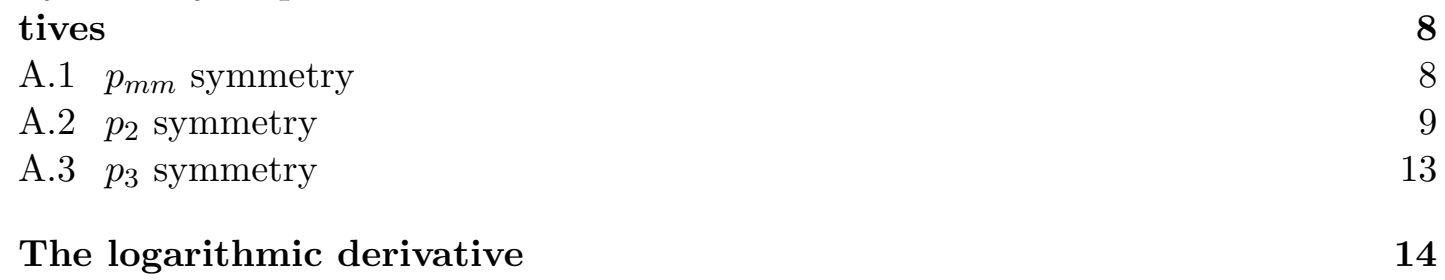


\begin{tabular}{lll}
\hline B.1 Symmetry-appropriate derivatives & 14
\end{tabular}

\begin{tabular}{lll}
\hline B.2 Gauge transformations and lattice translations & 16
\end{tabular}

\begin{tabular}{lll}
\hline B.3 & Real-valued Wannier functions & 16
\end{tabular}

\begin{tabular}{|ll|}
\hline B.4 The sign of the logarithmic derivative & 17
\end{tabular}

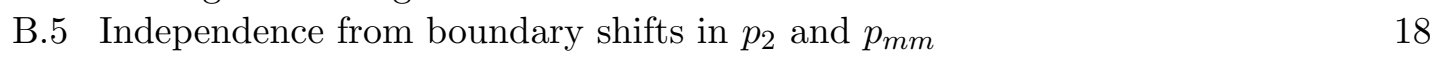

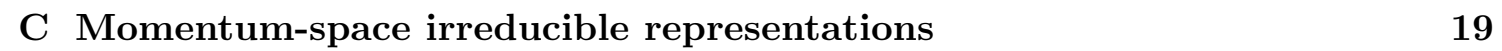

$\begin{array}{lll}\text { C.1 } & p_{2} \text {-symmetric atomic insulators } & 19\end{array}$

\begin{tabular}{lll}
\hline C.2 & $p_{m m}$-symmetric atomic insulators & 20 \\
\hline
\end{tabular}

\begin{tabular}{lr}
\hline References & 22
\end{tabular}

\section{Introduction}

Topological insulators are materials characterised by a bulk topological invariant, which predicts the existence of protected boundary modes localized at the materials' edges [1 -3$]$. This bulk-boundary correspondence has led to proposals for devices exploiting robust electronic, electromagnetic, and mechanical transport properties across a wide range of systems, from quantum materials, to cold atoms, and active classical matter [4 10]. Although there is no general proof for the correspondence between bulk topology and the presence of boundary modes [11], it is commonly accepted that topological insulators with a non-zero value for the so-called strong topological invariant host boundary states that are protected against back-scattering [3]. This is true both for systems with broken time-reversal symmetry whose strong invariant arises from the Chern numbers of its bands [2, 12, and for time-reversal symmetric systems, where the strong invariant is of the $\mathrm{Z}_{2}$, or Fu-Kane-Mele (FKM), type [15 17]. Both Chern numbers and FKM invariants emerge from a non-zero integrated Berry curvature in the bands of a topological insulator. It has recently been pointed out, however, that a complete classification of topological insulators takes into account the spatial symmetries of the lattice as well as the Berry curvature 18 20]. The relation between the symmetries of the electronic states in an insulator's Bloch bands and the symmetries of the underlying atomic lattice are encoded in band representations, which can act as (weak) topological invariants in their own right 1921 .

Here, we show that the band representations of two atomic insulators without timereversal, particle-hole, or chiral symmetries (class A) and with trivial strong topological invariants, can predict the emergence of boundary states localised at the interface between the materials when brought into a heterojunction geometry. This can be seen as a generalised bulk-boundary correspondence, in which the boundary signifies a transition from one material into any topologically distinct medium rather than necessarily connecting to the vacuum. The correspondence naturally complements the classification of topological insulators in terms of band representations, and agrees with its predictions [18]. The fact that the weak invariants in this correspondence are due to band representations, immediately implies that the topological interface states they give rise to will be protected by lattice symmetries only. Nevertheless, the recent progress in the controlled growth and manipulation of heterostructures [22 24], suggests a possible role for topological interface states in mesoscopic devices based on electronic transport properties.

For the sake of being specific, we focus below on two-dimensional systems of spinless fermions, and restrict the discussion to materials with only simple bands and elementary 
band representations. We detail the analysis of junctions joining two materials with equal space groups, being either p2, p3, or pmm. Based on these examples, we outline the general procedure for identifying topological interface states, and argue that such states generically arise in junctions of inversion symmetric materials.

\section{Bulk-boundary correspondence}

The allowed shapes of electronic wave functions in crystals are determined by the symmetries of its atomic lattice 25]. For atomic insulators with periodic boundary conditions, the real-space wave functions of so-called simple bands (which necessarily are also elementary band representations) 26 29, can be written in the Bloch-like form:

$$
\psi^{(\mathbf{w}, l)}(\mathbf{k} ; \mathbf{r})=\frac{1}{\Omega} \sum_{\mathbf{R}_{n}} e^{i \mathbf{k} \cdot \mathbf{R}_{n}} a^{(\mathbf{w}, l)}\left(\mathbf{r}-\mathbf{R}_{n}\right) .
$$

Here, $\mathbf{R}_{n}$ are the centres of the unit cells, while $\mathbf{w}$ is a Wyckoff position, i.e. a point inside the unit cell that is mapped onto itself (modulo lattice translations) by the point group of the crystal or one of its subgroups. The Wannier functions $a^{(\mathbf{w}, l)}$ are symmetry-appropriate basis functions centered at $\mathbf{w}$, which means that under the point group operations leaving $\mathbf{w}$ invariant, these functions transform as the irreducible representation labeled by $l$. Notice that the index $(\mathbf{w}, l)$ applies for any value of $\mathbf{k}$, and thus determines the real-space symmetry of the entire Bloch band [30]. These real-space labels are an alternative to labelling bands by a set of irreducible representations at high-symmetry points in momentum space, and for the p2 and pmm-symmetric atomic insulators considered here, there is a one-to-one relation between the two conventions 31 33 .

In crystals with open boundary conditions, bulk states become standing waves built from linear combinations of Bloch states with opposing momenta, with small deformations that allow them to smoothly connect to decaying vacuum solutions outside the crystal or to different standing waves in a neighbouring crystal $34-36$. For standing waves built from Bloch states at high-symmetry momenta, lattice symmetry alone may force either the real-space wave function or its derivative to be zero at Wyckoff positions, making it impossible to connect to the outside if the material terminates at such a position. The only way to overcome this obstruction, is for the Bloch momentum value to acquire an imaginary component, corresponding to an exponential decay inside the bulk of the crystal. In other words, whenever the existence of bulk states is prevented by lattice symmetry, an exponentially localised edge state arises [37]. Moreover, because the bulk standing waves are constructed from linear combinations of Bloch states, their matching conditions at the crystal's boundary can be written entirely in terms of Bloch state properties. There is thus a direct correspondence between properties of bulk states calculated with periodic boundary conditions, and the existence of edge or interface states in corresponding materials with boundaries.

The obstruction to constructing bulk states in strong topological insulators is signalled by the presence of non-zero net Berry curvature [38, 39]. In atomic insulators with nontrivial symmetry-based topological invariants $[18[20]$, an analogous obstruction may be formulated in terms of the symmetry labels $(\mathbf{w}, l)$ of occupied bands. For one-dimensional crystals with inversion symmetry, this bulk-boundary correspondence has been explicitly constructed [37, 40]. Here, we extend these results to two (and higher) dimensions, and show how they can be used to predict the presence of localised states at the interface joining two atomic insulators. 


\section{Interface states}

For electronic states to smoothly connect across an interface, the wave functions and their derivatives need to match on either side [34,41]. It has been shown that this smooth matching of standing waves in an open system is possible only if a similar smooth connection can be made using the Bloch waves of the corresponding periodic bulk [34 36]. This can be seen as an incarnation of the bulk-boundary correspondence, which allows us to predict the feasibility of matching wave functions in an open system by studying the shape of Bloch waves in the periodic setup. Moreover, if no obstruction for joining together bulk states exists, localised edge state solutions do not arise [37. To find topological edge states, it thus suffices to check whether Bloch states with complex momentum values can be smoothly connected across an interface. This is most conveniently done using the logarithmic derivative, defined as:

$$
\rho^{(\mathbf{w}, l)}(\mathbf{k}, \mathbf{r})=\frac{\mathbf{n} \cdot \nabla \psi^{(\mathbf{w}, l)}(\mathbf{k}, \mathbf{r})}{\psi^{(\mathbf{w}, l)}(\mathbf{k}, \mathbf{r})} .
$$

Here, $\mathbf{n}$ is a vector normal to the boundary. The logarithmic derivative is invariant under both gauge transformations (changing the Wannier basis) and translations by lattice vectors. Moreover, for any space group that includes inversion symmetry and allows for real-valued Wannier functions, such as $\mathrm{p} 2$ or pmm, $\rho(\mathbf{k}, \mathbf{r})$ can be shown to be purely imaginary for any real value of momentum, and purely real whenever the real part of $\mathbf{k}$ is a high-symmetry point in the Brillouin zone 40] (see Appendix B.3 for details).

This is particularly convenient in the search for boundary states, which arise only at high-symmetry momenta. That is, solutions to Schrödinger's equation with real $\mathbf{k}$ exist for any momentum value along the bulk of any band. At high-symmetry points, however, the lattice symmetry forces either the wave function or its derivative to vanish, and these conditions may pose an obstruction to creating smoothly connected states across the boundary. In that case, there will be an edge mode with $\mathbf{k}_{\text {edge }}=\mathbf{k}_{\mathrm{R}}+i \mathbf{k}_{\mathrm{I}}$, where $\mathbf{k}_{\mathrm{R}}$ is the high-symmetry momentum value. Since the logarithmic derivative for $\mathbf{k}_{\text {edge }}$ is purely real, an edge mode can only arise if the signs of $\rho\left(\mathbf{k}_{\text {edge }}, \mathbf{r}\right)$ agree on either side of the junction. Here, $\mathbf{r}$ denotes the location of the edge, which should coincide with a highsymmetry point of the lattice. In fact, the matching of signs has been shown to be both a necessary and sufficient condition [37].

For inversion-symmetric crystals, the lattice symmetry causes a zero in either the Bloch state or its derivative at any high-symmetry momentum, forcing $\rho(\mathbf{k}, \mathbf{r})$ to go to either zero or infinity [40]. Other, accidental zeroes or infinities always come in pairs, and may be ignored without loss of generality. Given the band label $(\mathbf{w}, l)$ and a location $\mathbf{r}$ along the crystal boundary (chosen to be a Wyckoff position), the zeroes and infinities of $\rho$ at highsymmetry momenta are entirely fixed by the crystal symmetry (as shown in Appendix A for $\mathrm{p} 2$, pmm, and $\mathrm{p} 3$ ). Together with the fact that Bloch functions can be analytically continued to complex momentum values [42 44], these zeroes and infinities in turn fix the sign of the logarithmic derivative for any putative boundary state 40 .

Consider for example the band with zeroes at both $\Gamma$ and $X$, and assume that $\rho$ starts off positive for boundary states near $\Gamma$, as shown schematically in the bottom left panel of Fig. 1 That is, for small $\epsilon$ a power series expansion of the logarithmic derivative yields $\rho(\Gamma-i \epsilon) \sim \epsilon$. This can be analytically continued to propagating bulk modes near $\Gamma$ by writing it as $\rho(\Gamma-z) \sim-i z$, which implies $\rho(\Gamma+\epsilon) \sim i \epsilon$. When traversing the band from $\Gamma$ to $X$, the logarithmic derivative has to stay purely imaginary. Moreover, it cannot pass through the origin since there are no zeroes or infinities outside of the high-symmetry momenta. We thus find that $\rho(X-\epsilon) \sim i \epsilon$ as well. This can again be 

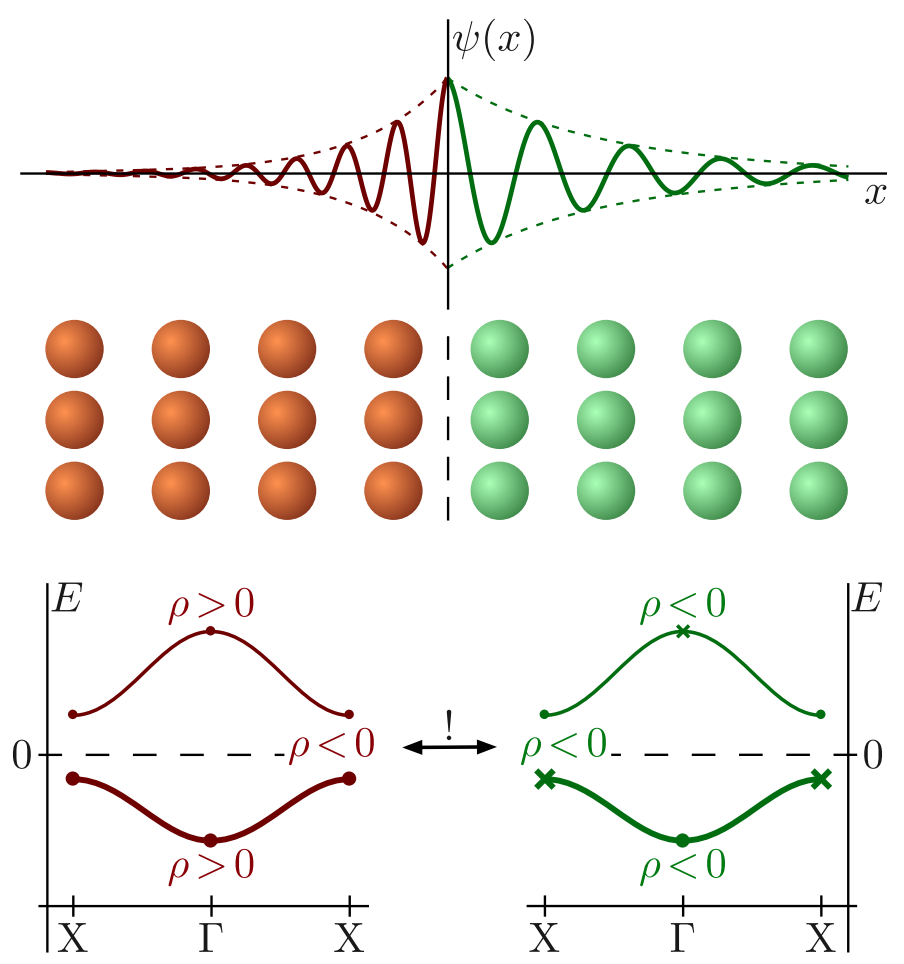

Figure 1: Topological mode at the interface of two atomic insulators. Exponentially localised states (top) may arise at the interface separating two inversion-symmetric atomic insulators (middle), if their individual band structures present a topological obstruction for bulk states to connect across the interface (bottom). The obstruction is diagnosed by the real-space symmetry labels of the occupied bands (thick lines), which uniquely determine the zeroes of the Bloch wave function (solid dots) and its derivative (crosses) at high-symmetry points in the Brillouin zone. These in turn yield the sign of the logarithmic derivative $\rho$ in any of the bulk energy gaps. Whenever the sign is the same for gaps at equal energies at either side of the interface, a localised topological interface state emerges.

analytically continued toward boundary states near $X$ by first writing $\rho(X-z) \sim i z$, and thus $\rho(X-i \epsilon) \sim-\epsilon<0$. A band connecting two zeroes thus causes a change in sign of the logarithmic derivative when going from one band gap to the next (Fig. 1. bottom left panel). If instead there had been an infinity at $X$, we would have found $\rho(X-\epsilon) \sim i / \epsilon$, and thus $\rho(X-i \epsilon) \sim 1 / \epsilon>0$ (Fig. 1. bottom right). Continuing in this way, we find that $\rho$ changes sign across a band with either two zeroes of two infinities, and stays fixed otherwise. All signs of $\rho$ are thus fixed by the lattice symmetry once the band labels $(\mathbf{w}, l)$ are given.

In a heterojunction of two atomic insulators, a boundary state localised at the interface will arise whenever the two materials have a band gap over the same range of energies, and the logarithmic derivatives on either side of the junction have the same sign for some complex $\mathbf{k}=\mathbf{k}_{\mathrm{R}}+i \mathbf{k}_{\mathrm{I}}$, with $\mathbf{k}_{\mathrm{R}}$ a high-symmetry point. Since the symmetry labels of the bands in p2 and pmm-symmetric crystals fully determine the sign changes of $\rho$ on either side of the junction, they also predict in which band gaps interface states will form, as shown schematically in the example of Fig. 1.

Notice that this argument requires both inversion symmetry and the existence of realvalued Wannier states to relate the effect of symmetry operations to that of complex 
conjugation. The three-fold rotation in p3, for example, can have eigenvalues $e^{ \pm i 2 \pi / 3}$, which cannot be realised in any real-valued Wannier state. For bands characterised by such eigenvalues, the real-space symmetry operations still impose constraints on the logarithmic derivative, for example relating the signs along symmetry-related edges (as shown in Appendix A.3 for p3), but in general, these do not sufficiently restrict $\rho$ to predict the presence or absence of interface states. Similarly, the absence of inversion symmetry in p3 also prevents the prediction of interface states for its real-valued Wannier states.

\section{Topology of interface states}

Interface states between crystals with different symmetry labels occurring at the Fermi level $\left(E_{\mathrm{F}}\right)$ are expected to be topological in nature, based on the classification of topological insulators with crystalline symmetry [18]. Since topological phase transitions by definition involve either the closing of the band gap at the Fermi level or a change of lattice symmetry, smooth deformations of the Hamiltonian that do neither of these things should have no effect on the interface states. To see that this is indeed the case for the interface states discussed here, notice that the analysis predicting their presence at $E_{\mathrm{F}}$ depends only on the band symmetry labels on either side of the junction 1 . The only deformations of the Hamiltonian that can affect the interface states then, are changes in the symmetry labels. These are caused by band inversions at high-symmetry points in momentum space.

In atomic insulators it is always possible to induce the momentum space representations at high-symmetry points from the real-space band labels [32, 45]. The former are more general, in the sense that they describe strong topological insulators with non-zero Chern numbers as well as atomic insulators, whereas the real-space band symmetry labels can be applied only to atomic insulators $19,30,39$. For crystals with p2 or pmm symmetry, the mapping from real space to momentum space labels is especially straightforward. For these groups, the lattice symmetry causes either the Bloch state or its derivative to go to zero at all high symmetry momenta, depending on the band symmetry label. At the same time, a Bloch state can only have zero slope if it is even under reflection, and have zero value (but not zero slope) if it is odd. The zeros and infinities of the logarithmic derivative $\rho$ thus indicate how the Bloch state transforms under inversion at high-symmetry points, or under mirror operations along high-symmetry lines. Together with consistency requirements throughout the Brillouin zone, this completely fixes the momentum-space representation (see Appendix $\mathrm{C}$ for details).

A band inversion at a high-symmetry point can be regarded as the exchange of momentum-space representations between two bands at that point. Because for $\mathrm{p} 2$ and pmm the representations entirely determine the value of $\rho$, the band inversion can equivalently be described as an exchange of the zeroes or infinities of $\rho$ at the high-symmetry point (see Fig. 2). As shown before, the sign of $\rho$ for edge states associated with a particular high-symmetry point $\mathbf{k}$ can be determined based on the sign of $\rho$ in a lower-energy band gap associated with a different point $\mathbf{k}^{\prime}$ (recall Fig. 1). If the line connecting $\mathbf{k}^{\prime}$ and $\mathbf{k}$ has either two zeroes or two infinities at its end points, the sign of $\rho$ changes from one gap to the next. For one zero and one infinity, the signs are equal. If we now consider a succession of two bands, as in Fig. 2, interchanging the zeroes or infinities in the middle can be easily seen not to affect the relation between the sign of $\rho$ below the lowest and above the highest band. Either two bands with no sign flips transform into two bands

\footnotetext{
${ }^{1}$ Strictly speaking, it also depends on the sign of $\rho$ for energies below the lowest band, which is not determined by the symmetry labels. This sign, however, is fixed by the physical requirement that there are no edge states below the lowest occupied band.
} 

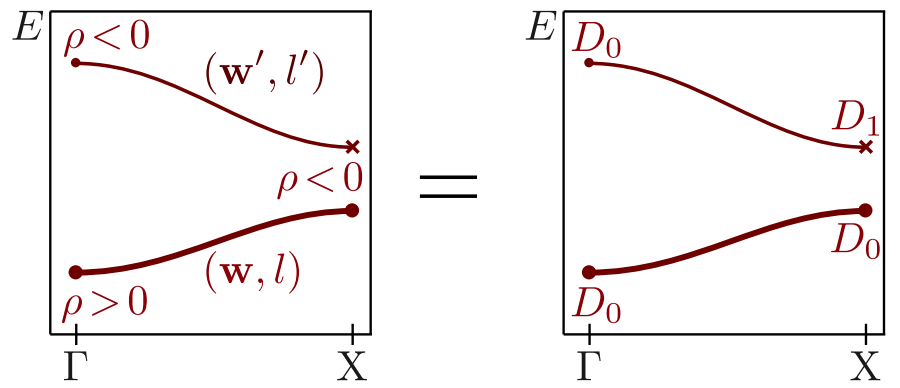

inversion at $\mathrm{X}$
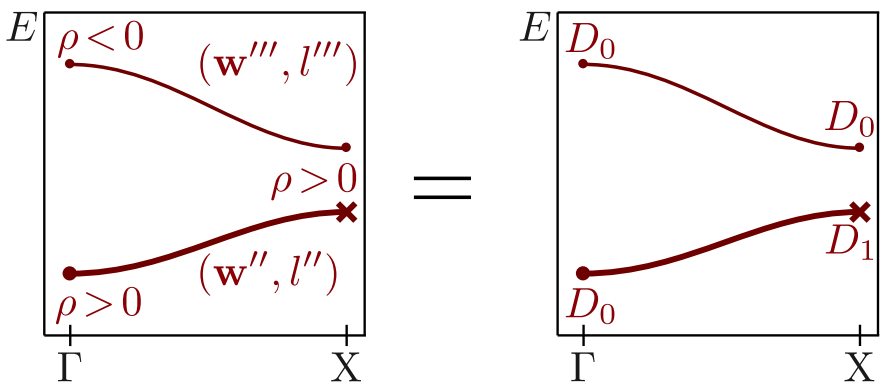

Figure 2: The effect of a band inversion. In inversion-symmetric atomic insulators, the real-space symmetry labels $(\mathbf{w}, l)$ that determine the sign of the logarithmic derivative in bulk energy gaps (top left), also fix the representations of the space group symmetries relevant to high-symmetry points in the Brillouin zone (top right). A band inversion implies the exchange of representations across an energy gap at one or several of the highsymmetry points (bottom right). This may result in the logarithmic derivative $\rho$ changing sign in that gap, and in the symmetry-labels of adjacent bands being altered, but it does not affect the signs of $\rho$ in any other bulk energy gaps (bottom left).

that both flip the sign of $\rho$ (or the other way around), or a band with a sign flip changes places with a band without. As long as only occupied bands undergo band inversions, the relation between the sign of $\rho$ in the topmost gap, at the Fermi level, and the sign at the bottom, below all occupied bands (which is fixed) thus stays constant. That is, the sign of $\rho$ at $E_{\mathrm{F}}$, and hence the presence of interface states, can only be changed in a topological phase transition, involving the closing of the gap between valence and conductance bands or a change in lattice symmetry.

The correspondence of the topology of interface states predicted by real-space band symmetry labels and the momentum-space classification of crystalline topological insulators can be made by realising that band inversions which exchange zeroes and infinities of $\rho$ at high-symmetry momenta are accompanied by the exchange of representations at those points. This does not change the total number of bands in any given representation at a high-symmetry point, which was found to be the most general topological invariant for crystalline topological insulators [18 20]. The band inversions that do not affect the sign of $\rho$ at $E_{\mathrm{F}}$, and thus leave the edge states fixed, are also precisely the transformations of the Hamiltonian that do not change its topological classification. 


\section{Discussion}

We have shown that crystalline topological insulators may harbour topologically protected edge states, even in the atomic limit. These edge states are not associated with any Berry curvature or intrinsic symmetry, but rather rely on the symmetries of the atomic lattice itself. We have shown that these states arise naturally at the interface between twodimensional atomic insulators with inversion symmetry and real-valued Wannier states, which may be realised in heterojunction architectures. The inversion symmetry protecting the interface states can be realised in two dimensions either as a two-fold rotation or as the product of two mirror operations. In higher dimensions, the same arguments are expected to apply in the presence of any symmetries relating states at $\mathbf{k}$ and $\mathbf{- k}$. These could be three mirror planes, combinations of mirror planes and rotation axes, or a threedimensional inversion centre. The interface states are topologically robust in the sense that they cannot be removed by smooth deformations of the Hamiltonian that do not close the conductance gap or change the symmetry of the atomic lattice.

Since the interface states are protected by lattice symmetry, their existence does in general depend sensitively on the terminations of the crystals on either side of the junction. This sensitivity may disappear, however, in a special type of heterostructure, with the same atomic lattice on either side. Such interfaces may arise for example when inhomogeneous doping or external fields are applied to a single crystal, causing phase separation without affecting the atomic structure. In Appendix B.5, it is shown explicitly that in such cases, with $\mathrm{p} 2$ or pmm symmetry, shifting the location of the interface always affects the sign of $\rho$ on both sides of the junction in the same way, leaving their product invariant. For this special type of heterojunction, the existence of interface states is robust not only to band inversions, but also to changes and defects in the interface geometry.

Author contributions All authors contributed to all stages of the research.

\section{Appendices}

\section{A Symmetry-imposed zeroes of Bloch wave functions and their derivatives}

\section{A.1 $p_{m m}$ symmetry}

In a crystal with $p_{m m}$ space group symmetry, there are four inequivalent Wyckoff positions [46]. Each of these are left invariant by the symmetry operations of the point group $D_{2}$, whose characters are given in table 1 [25]. The non-trivial group operations are reflections $(x, y) \rightarrow(-x, y)$ and $(x, y) \rightarrow(x,-y)$, written $M_{x}$ and $M_{y}$, and their product, which is equivalent to a $180^{\circ}$ rotations around an axis perpendicular to the plane and is therefore denoted $C_{2}$. For each of the four Wyckoff positions $\mathbf{w}$, we can define a phase factor associated with the symmetry operation $\alpha$ as $\mathbf{R}_{\mathrm{w}}^{\alpha}=\mathbf{w}-\alpha \mathbf{w} 31$. The phase factors for the non-trivial operations in the $D_{2}$ point group are listed in table 2.

For each of the non-trivial point group operations $\alpha$, the wave function $\psi^{(\mathbf{w}, l)}$, built from Wannier functions centered at Wyckoff position $w$ and transforming as representation $D_{l}$ of the point group, is constrained by the following relations [31]:

$$
\begin{aligned}
\alpha \psi^{(\mathbf{w}, l)}(\mathbf{k} ; \mathbf{r}) & =\psi^{(\mathbf{w}, l)}\left(\alpha^{-1} \mathbf{k} ; \alpha^{-1} \mathbf{r}\right) \\
& =e^{i \mathbf{k} \cdot \mathbf{R}_{\mathbf{w}}^{\alpha}} D_{l}(\alpha) \psi^{(\mathbf{w}, l)}(\mathbf{k} ; \mathbf{r}) .
\end{aligned}
$$


Table 1: Character table for the point group $D_{2}$, relevant at any of the Wyckoff positions in a $p_{m m}$-symmetric atomic insulator. $E$ labels the identity operation.

\begin{tabular}{rrrrr}
\hline \hline & $E$ & $M_{x}$ & $M_{y}$ & $C_{2}$ \\
\hline$D_{0}$ & 1 & 1 & 1 & 1 \\
$D_{1}$ & 1 & -1 & -1 & 1 \\
$D_{2}$ & 1 & 1 & -1 & -1 \\
$D_{3}$ & 1 & -1 & 1 & -1 \\
\hline
\end{tabular}

Table 2: The phase factor associated with the non-trivial $D_{2}$ point group operations for the four Wyckoff positions in a $p_{m m}$-symmetric atomic insulator.

\begin{tabular}{crrr}
\hline \hline $\mathbf{w}$ & $\mathbf{R}_{\mathbf{w}}^{M_{x}}$ & $\mathbf{R}_{\mathbf{w}}^{M_{y}}$ & $\mathbf{R}_{\mathbf{w}}^{C_{2}}$ \\
\hline $\mathbf{w}_{a}=(0,0)$ & $\mathbf{0}$ & $\mathbf{0}$ & $\mathbf{0}$ \\
$\mathbf{w}_{b}=\left(\frac{a}{2}, 0\right)$ & $a \mathbf{e}_{x}$ & $\mathbf{0}$ & $a \mathbf{e}_{x}$ \\
$\mathbf{w}_{c}=\left(0, \frac{a}{2}\right)$ & $\mathbf{0}$ & $a \mathbf{e}_{y}$ & $a \mathbf{e}_{y}$ \\
$\mathbf{w}_{d}=\left(\frac{a}{2}, \frac{a}{2}\right)$ & $a \mathbf{e}_{x}$ & $a \mathbf{e}_{y}$ & $a \mathbf{e}_{x}+a \mathbf{e}_{y}$
\end{tabular}

Taking both $\mathbf{k}$ and $\mathbf{r}$ to be invariant under the operation of $\alpha$, these conditions can be met only if either $e^{i \mathbf{k} \cdot \mathbf{R}_{\mathbf{w}}^{\alpha}} D_{l}(\alpha)=1$, or the wave function is zero.

For example, the symmetry operation $M_{x}$ leaves invariant any k-point on the lines $\mathbf{\Gamma Y}$ and XM (up to translations by a reciprocal lattice vector), and any real-space point on lines $x=0$ and $x=a / 2$ (modulo translations by a lattice vector). For a crystal terminating at a boundary with $x=0$, we find $\mathbf{R}_{\mathbf{w}}^{M_{x}}=0$, so that any band transforming as either $D_{1}$ or $D_{3}$ will be forced to have its wave function go to zero on the lines $\mathbf{\Gamma Y}$ and $\mathbf{X M}$ in the Brillouin zone. The symmetry is thus particularly important for crystals terminating at a boundary with fixed $x$ coordinate, in which symmetry imposes the suppression of the wave function and hence the appearance of an edge state along an entire boundary.

Besides imposing zeroes on the Bloch wave function, the symmetry may also impose zeroes on the directional derivatives of the Bloch wave function. For example, if a Bloch wave function is even under the reflection $(x, y) \rightarrow(-x, y)$, then its normal derivative along $x$ is necessarily odd under the same reflections. That is, if the wave function transforms as $D_{0}$ or $D_{2}$, its normal derivative along $x$ will transform as respectively $D_{3}$ or $D_{1}$, and vice versa. Equation (3) then imposes zeroes on the normal derivatives in the same way as it does for the Bloch wave function itself.

Tables 3 through 6 list the symmetry-imposed zeroes in both the Bloch wave function and its normal derivatives for all possible combinations of high-symmetry positions in real and reciprocal space.

\section{A.2 $p_{2}$ symmetry}

Crystals with $p_{2}$ space group symmetry, again have four inequivalent Wyckoff positions, $(0,0),(a / 2,0),(0, a / 2)$, and $(a / 2, a / 2)$ [46], and four high symmetry points in the Brillouin Zone, $\boldsymbol{\Gamma}, \mathbf{X}, \mathbf{Y}$, and $\mathbf{M}$. They are all left invariant by the elements of the point group $C_{2}$, whose character table 25] and phase factors $e^{i \mathbf{k} \cdot \mathbf{R}_{\mathrm{w}}^{C_{2}}}$ are provided in table 7. Applying equation (3) again yields symmetry-enforced zeroes, listed in table 8. 
Table 3: Zeroes imposed by $p_{m m}$-symmetry on either the Bloch wave function or its normal derivative along $\hat{\mathbf{e}}_{x}$. The real-space positions are fixed at $\mathbf{r}=(0, y)$, for arbitrary values of the $y$ coordinate.

\begin{tabular}{rrrrrr}
\hline \hline$(w, l), \mathbf{r}$ & $\mathbf{k}=\mathbf{\Gamma}$ & $\mathbf{X}$ & $\mathbf{Y}$ & $\mathbf{M}$ \\
\hline$\left(\mathbf{w}_{a}, 0\right),(0, y)$ & $\partial_{x} \psi=0$ & $\partial_{x} \psi=0$ & $\partial_{x} \psi=0$ & $\partial_{x} \psi=0$ \\
$\left(\mathbf{w}_{a}, 1\right),(0, y)$ & $\psi=0$ & $\psi=0$ & $\psi=0$ & $\psi=0$ \\
$\left(\mathbf{w}_{a}, 2\right),(0, y)$ & $\partial_{x} \psi=0$ & $\partial_{x} \psi=0$ & $\partial_{x} \psi=0$ & $\partial_{x} \psi=0$ \\
$\left(\mathbf{w}_{a}, 3\right),(0, y)$ & $\psi=0$ & $\psi=0$ & $\psi=0$ & $\psi=0$ \\
$\left(\mathbf{w}_{b}, 0\right),(0, y)$ & $\partial_{x} \psi=0$ & $\psi=0$ & $\partial_{x} \psi=0$ & $\psi=0$ \\
$\left(\mathbf{w}_{b}, 1\right),(0, y)$ & $\psi=0$ & $\partial_{x} \psi=0$ & $\psi=0$ & $\partial_{x} \psi=0$ \\
$\left(\mathbf{w}_{b}, 2\right),(0, y)$ & $\partial_{x} \psi=0$ & $\psi=0$ & $\partial_{x} \psi=0$ & $\psi=0$ \\
$\left(\mathbf{w}_{b}, 3\right),(0, y)$ & $\psi=0$ & $\partial_{x} \psi=0$ & $\psi=0$ & $\partial_{x} \psi=0$ \\
$\left(\mathbf{w}_{c}, 0\right),(0, y)$ & $\partial_{x} \psi=0$ & $\partial_{x} \psi=0$ & $\partial_{x} \psi=0$ & $\partial_{x} \psi=0$ \\
$\left(\mathbf{w}_{c}, 1\right),(0, y)$ & $\psi=0$ & $\psi=0$ & $\psi=0$ & $\psi=0$ \\
$\left(\mathbf{w}_{c}, 2\right),(0, y)$ & $\partial_{x} \psi=0$ & $\partial_{x} \psi=0$ & $\partial_{x} \psi=0$ & $\partial_{x} \psi=0$ \\
$\left(\mathbf{w}_{c}, 3\right),(0, y)$ & $\psi=0$ & $\psi=0$ & $\psi=0$ & $\psi=0$ \\
$\left(\mathbf{w}_{d}, 0\right),(0, y)$ & $\partial_{x} \psi=0$ & $\psi=0$ & $\partial_{x} \psi=0$ & $\psi=0$ \\
$\left(\mathbf{w}_{d}, 1\right),(0, y)$ & $\psi=0$ & $\partial_{x} \psi=0$ & $\psi=0$ & $\partial_{x} \psi=0$ \\
$\left(\mathbf{w}_{d}, 2\right),(0, y)$ & $\partial_{x} \psi=0$ & $\psi=0$ & $\partial_{x} \psi=0$ & $\partial_{x} \psi=0$ \\
$\left(\mathbf{w}_{d}, 3\right),(0, y)$ & $\psi=0$ & $\partial_{x} \psi=0$ & $\psi=0$ & $\partial_{x} \psi=0$ \\
\hline
\end{tabular}

Table 4: Zeroes imposed by $p_{m m}$-symmetry on either the Bloch wave function or its normal derivative along $\hat{\mathbf{e}}_{x}$. The real-space positions are fixed at $\mathbf{r}=(a / 2, y)$, for arbitrary values of the $y$ coordinate.

\begin{tabular}{rrrrrr}
\hline \hline$(w, l), \mathbf{r}$ & $\mathbf{k}=\mathbf{\Gamma}$ & $\mathbf{X}$ & $\mathbf{Y}$ & $\mathbf{M}$ \\
\hline$\left(\mathbf{w}_{a}, 0\right),\left(\frac{a}{2}, y\right)$ & $\partial_{x} \psi=0$ & $\psi=0$ & $\partial_{x} \psi=0$ & $\psi=0$ \\
$\left(\mathbf{w}_{a}, 1\right),\left(\frac{a}{2}, y\right)$ & $\psi=0$ & $\partial_{x} \psi=0$ & $\psi=0$ & $\partial_{x} \psi=0$ \\
$\left(\mathbf{w}_{a}, 2\right),\left(\frac{a}{2}, y\right)$ & $\partial_{x} \psi=0$ & $\psi=0$ & $\partial_{x} \psi=0$ & $\psi=0$ \\
$\left(\mathbf{w}_{a}, 3\right),\left(\frac{a}{2}, y\right)$ & $\psi=0$ & $\partial_{x} \psi=0$ & $\psi=0$ & $\partial_{x} \psi=0$ \\
$\left(\mathbf{w}_{b}, 0\right),\left(\frac{a}{2}, y\right)$ & $\partial_{x} \psi=0$ & $\partial_{x} \psi=0$ & $\partial_{x} \psi=0$ & $\partial_{x} \psi=0$ \\
$\left(\mathbf{w}_{b}, 1\right),\left(\frac{a}{2}, y\right)$ & $\psi=0$ & $\psi=0$ & $\psi=0$ & $\psi=0$ \\
$\left(\mathbf{w}_{b}, 2\right),\left(\frac{a}{2}, y\right)$ & $\partial_{x} \psi=0$ & $\partial_{x} \psi=0$ & $\partial_{x} \psi=0$ & $\partial_{x} \psi=0$ \\
$\left(\mathbf{w}_{b}, 3\right),\left(\frac{a}{2}, y\right)$ & $\psi=0$ & $\psi=0$ & $\psi=0$ & $\psi=0$ \\
$\left(\mathbf{w}_{c}, 0\right),\left(\frac{a}{2}, y\right)$ & $\partial_{x} \psi=0$ & $\psi=0$ & $\partial_{x} \psi=0$ & $\psi=0$ \\
$\left(\mathbf{w}_{c}, 1\right),\left(\frac{a}{2}, y\right)$ & $\psi=0$ & $\partial_{x} \psi=0$ & $\psi=0$ & $\partial_{x} \psi=0$ \\
$\left(\mathbf{w}_{c}, 2\right),\left(\frac{a}{2}, y\right)$ & $\partial_{x} \psi=0$ & $\psi=0$ & $\partial_{x} \psi=0$ & $\psi=0$ \\
$\left(\mathbf{w}_{c}, 3\right),\left(\frac{a}{2}, y\right)$ & $\psi=0$ & $\partial_{x} \psi=0$ & $\psi=0$ & $\partial_{x} \psi=0$ \\
$\left(\mathbf{w}_{d}, 0\right),\left(\frac{a}{2}, y\right)$ & $\partial_{x} \psi=0$ & $\partial_{x} \psi=0$ & $\partial_{x} \psi=0$ & $\partial_{x} \psi=0$ \\
$\left(\mathbf{w}_{d}, 1\right),\left(\frac{a}{2}, y\right)$ & $\psi=0$ & $\psi=0$ & $\psi=0$ & $\psi=0$ \\
$\left(\mathbf{w}_{d}, 2\right),\left(\frac{a}{2}, y\right)$ & $\partial_{x} \psi=0$ & $\partial_{x} \psi=0$ & $\partial_{x} \psi=0$ & $\partial_{x} \psi=0$ \\
$\left(\mathbf{w}_{d}, 3\right),\left(\frac{a}{2}, y\right)$ & $\psi=0$ & $\psi=0$ & $\psi=0$ & $\psi=0$ \\
\hline
\end{tabular}


Table 5: Zeroes imposed by $p_{m m}$-symmetry on either the Bloch wave function or its normal derivative along $\hat{\mathbf{e}}_{y}$. The real-space positions are fixed at $\mathbf{r}=(x, 0)$, for arbitrary values of the $x$ coordinate.

\begin{tabular}{rrrrrr}
\hline \hline$(w, l), \mathbf{r}$ & $\mathbf{k}=\mathbf{\Gamma}$ & $\mathbf{X}$ & $\mathbf{Y}$ & $\mathbf{M}$ \\
\hline$\left(\mathbf{w}_{a}, 0\right),(x, 0)$ & $\partial_{y} \psi=0$ & $\partial_{y} \psi=0$ & $\partial_{y} \psi=0$ & $\partial_{y} \psi=0$ \\
$\left(\mathbf{w}_{a}, 1\right),(x, 0)$ & $\psi=0$ & $\psi=0$ & $\psi=0$ & $\psi=0$ \\
$\left(\mathbf{w}_{a}, 2\right),(x, 0)$ & $\psi=0$ & $\psi=0$ & $\psi=0$ & $\psi=0$ \\
$\left(\mathbf{w}_{a}, 3\right),(x, 0)$ & $\partial_{y} \psi=0$ & $\partial_{y} \psi=0$ & $\partial_{y} \psi=0$ & $\partial_{y} \psi=0$ \\
$\left(\mathbf{w}_{b}, 0\right),(x, 0)$ & $\partial_{y} \psi=0$ & $\partial_{y} \psi=0$ & $\partial_{y} \psi=0$ & $\partial_{y} \psi=0$ \\
$\left(\mathbf{w}_{b}, 1\right),(x, 0)$ & $\psi=0$ & $\psi=0$ & $\psi=0$ & $\psi=0$ \\
$\left(\mathbf{w}_{b}, 2\right),(x, 0)$ & $\psi=0$ & $\psi=0$ & $\psi=0$ & $\psi=0$ \\
$\left(\mathbf{w}_{b}, 3\right),(x, 0)$ & $\partial_{y} \psi=0$ & $\partial_{y} \psi=0$ & $\partial_{y} \psi=0$ & $\partial_{y} \psi=0$ \\
$\left(\mathbf{w}_{c}, 0\right),(x, 0)$ & $\partial_{y} \psi=0$ & $\partial_{y} \psi=0$ & $\psi=0$ & $\psi=0$ \\
$\left(\mathbf{w}_{c}, 1\right),(x, 0)$ & $\psi=0$ & $\psi=0$ & $\partial_{y} \psi=0$ & $\partial_{y} \psi=0$ \\
$\left(\mathbf{w}_{c}, 2\right),(x, 0)$ & $\psi=0$ & $\psi=0$ & $\partial_{y} \psi=0$ & $\partial_{y} \psi=0$ \\
$\left(\mathbf{w}_{c}, 3\right),(x, 0)$ & $\partial_{y} \psi=0$ & $\partial_{y} \psi=0$ & $\psi=0$ & $\psi=0$ \\
$\left(\mathbf{w}_{d}, 0\right),(x, 0)$ & $\partial_{y} \psi=0$ & $\partial_{y} \psi=0$ & $\psi=0$ & $\psi=0$ \\
$\left(\mathbf{w}_{d}, 1\right),(x, 0)$ & $\psi=0$ & $\psi=0$ & $\partial_{y} \psi=0$ & $\partial_{y} \psi=0$ \\
$\left(\mathbf{w}_{d}, 2\right),(x, 0)$ & $\psi=0$ & $\psi=0$ & $\partial_{y} \psi=0$ & $\partial_{y} \psi=0$ \\
$\left(\mathbf{w}_{d}, 3\right),(x, 0)$ & $\partial_{y} \psi=0$ & $\partial_{y} \psi=0$ & $\psi=0$ & $\psi=0$ \\
\hline
\end{tabular}

Table 6: Zeroes imposed by $p_{m m}$-symmetry on either the Bloch wave function or its normal derivative along $\hat{\mathbf{e}}_{y}$. The real-space positions are fixed at $\mathbf{r}=(x, a / 2)$, for arbitrary values of the $x$ coordinate.

\begin{tabular}{rrrrrr}
\hline \hline$(w, l), \mathbf{r}$ & $\mathbf{k}=\mathbf{\Gamma}$ & $\mathbf{X}$ & $\mathbf{Y}$ & $\mathbf{M}$ \\
\hline$\left(\mathbf{w}_{a}, 0\right),\left(x, \frac{a}{2}\right)$ & $\partial_{y} \psi=0$ & $\partial_{y} \psi=0$ & $\psi=0$ & $\psi=0$ \\
$\left(\mathbf{w}_{a}, 1\right),\left(x, \frac{a}{2}\right)$ & $\psi=0$ & $\psi=0$ & $\partial_{y} \psi=0$ & $\partial_{y} \psi=0$ \\
$\left(\mathbf{w}_{a}, 2\right),\left(x, \frac{a}{2}\right)$ & $\psi=0$ & $\psi=0$ & $\partial_{y} \psi=0$ & $\partial_{y} \psi=0$ \\
$\left(\mathbf{w}_{a}, 3\right),\left(x, \frac{a}{2}\right)$ & $\partial_{y} \psi=0$ & $\partial_{y} \psi=0$ & $\psi=0$ & $\psi=0$ \\
$\left(\mathbf{w}_{b}, 0\right),\left(x, \frac{a}{2}\right)$ & $\partial_{y} \psi=0$ & $\partial_{y} \psi=0$ & $\psi=0$ & $\psi=0$ \\
$\left(\mathbf{w}_{b}, 1\right),\left(x, \frac{a}{2}\right)$ & $\psi=0$ & $\psi=0$ & $\partial_{y} \psi=0$ & $\partial_{y} \psi=0$ \\
$\left(\mathbf{w}_{b}, 2\right),\left(x, \frac{a}{2}\right)$ & $\psi=0$ & $\psi=0$ & $\partial_{y} \psi=0$ & $\partial_{y} \psi=0$ \\
$\left(\mathbf{w}_{b}, 3\right),\left(x, \frac{a}{2}\right)$ & $\partial_{y} \psi=0$ & $\partial_{y} \psi=0$ & $\psi=0$ & $\psi=0$ \\
$\left(\mathbf{w}_{c}, 0\right),\left(x, \frac{a}{2}\right)$ & $\partial_{y} \psi=0$ & $\partial_{y} \psi=0$ & $\partial_{y} \psi=0$ & $\partial_{y} \psi=0$ \\
$\left(\mathbf{w}_{c}, 1\right),\left(x, \frac{a}{2}\right)$ & $\psi=0$ & $\psi=0$ & $\psi=0$ & $\psi=0$ \\
$\left(\mathbf{w}_{c}, 2\right),\left(x, \frac{a}{2}\right)$ & $\psi=0$ & $\psi=0$ & $\psi=0$ & $\psi=0$ \\
$\left(\mathbf{w}_{c}, 3\right),\left(x, \frac{a}{2}\right)$ & $\partial_{y} \psi=0$ & $\partial_{y} \psi=0$ & $\partial_{y} \psi=0$ & $\partial_{y} \psi=0$ \\
$\left(\mathbf{w}_{d}, 0\right),\left(x, \frac{a}{2}\right)$ & $\partial_{y} \psi=0$ & $\partial_{y} \psi=0$ & $\partial_{y} \psi=0$ & $\partial_{y} \psi=0$ \\
$\left(\mathbf{w}_{d}, 1\right),\left(x, \frac{a}{2}\right)$ & $\psi=0$ & $\psi=0$ & $\psi=0$ & $\psi=0$ \\
$\left(\mathbf{w}_{d}, 2\right),\left(x, \frac{a}{2}\right)$ & $\psi=0$ & $\psi=0$ & $\psi=0$ & $\psi=0$ \\
$\left(\mathbf{w}_{d}, 3\right),\left(x, \frac{a}{2}\right)$ & $\partial_{y} \psi=0$ & $\partial_{y} \psi=0$ & $\partial_{y} \psi=0$ & $\partial_{y} \psi=0$ \\
\hline
\end{tabular}


Table 7: The character table and phase factors associated with the point group $C_{2}$, relevant at Wyckoff positions in a $p_{2}$-symmetric atomic insulator.

\begin{tabular}{rrr}
\hline \hline & $E$ & $C_{2}$ \\
\hline$D_{0}$ & 1 & 1 \\
$D_{1}$ & 1 & -1 \\
\hline
\end{tabular}

\begin{tabular}{cr}
\hline \hline $\mathbf{w}$ & $\mathbf{R}_{\mathbf{w}}^{C_{2}}$ \\
\hline $\mathbf{w}_{a}=(0,0)$ & $\mathbf{0}$ \\
$\mathbf{w}_{b}=\left(\frac{a}{2}, 0\right)$ & $a \mathbf{e}_{x}$ \\
$\mathbf{w}_{c}=\left(0, \frac{a}{2}\right)$ & $a \mathbf{e}_{y}$ \\
$\mathbf{w}_{d}=\left(\frac{a}{2}, \frac{a}{2}\right)$ & $a \mathbf{e}_{x}+a \mathbf{e}_{y}$
\end{tabular}

Table 8: Zeroes of the Bloch wave function $\psi$ and its normal derivative $\psi^{\prime}$ in any direction, listed for all high-symmetry points in the Brillouin zone and real space.

\begin{tabular}{|c|c|c|c|c|c|}
\hline$(w, l), \mathbf{r}$ & $\mathrm{k}$ & $=\Gamma$ & $\overline{\mathrm{X}}$ & $\overline{\mathbf{Y}}$ & $\overline{\mathrm{M}}$ \\
\hline$\left(\mathbf{w}_{a}, 0\right),(0,0)$ & & $\psi^{\prime}=0$ & $\psi^{\prime}=0$ & $\psi^{\prime}=0$ & $\psi^{\prime}=0$ \\
\hline$\left(\mathbf{w}_{a}, 1\right),(0,0)$ & & $\psi=0$ & $\psi=0$ & $\psi=0$ & $\psi=0$ \\
\hline$\left(\mathbf{w}_{b}, 0\right),(0,0)$ & & $\psi^{\prime}=0$ & $\psi=0$ & $\psi^{\prime}=0$ & $\psi=0$ \\
\hline$\left(\mathbf{w}_{b}, 1\right),(0,0)$ & & $\psi=0$ & $\psi^{\prime}=0$ & $\psi=0$ & $\psi^{\prime}=0$ \\
\hline$\left(\mathbf{w}_{c}, 0\right),(0,0)$ & & $\psi^{\prime}=0$ & $\psi^{\prime}=0$ & $\psi=0$ & $\psi=0$ \\
\hline$\left(\mathbf{w}_{c}, 1\right),(0,0)$ & & $\psi=0$ & $\psi=0$ & $\psi^{\prime}=0$ & $\psi^{\prime}=0$ \\
\hline$\left(\mathbf{w}_{d}, 0\right),(0,0)$ & & $\psi^{\prime}=0$ & $\psi=0$ & $\psi=0$ & $\psi^{\prime}=0$ \\
\hline$\left(\mathbf{w}_{d}, 1\right),(0,0)$ & & $\psi=0$ & $\psi^{\prime}=0$ & $\psi^{\prime}=0$ & $\psi=0$ \\
\hline$\left(\mathbf{w}_{a}, 0\right),\left(\frac{a}{2}, 0\right)$ & & $\psi^{\prime}=0$ & $\psi=0$ & $\psi^{\prime}=0$ & $\psi=0$ \\
\hline$\left(\mathbf{w}_{a}, 1\right),\left(\frac{a}{2}, 0\right)$ & & $\psi=0$ & $\psi^{\prime}=0$ & $\psi=0$ & $\psi^{\prime}=0$ \\
\hline$\left(\mathbf{w}_{b}, 0\right),\left(\frac{a}{2}, 0\right)$ & & $\psi^{\prime}=0$ & $\psi^{\prime}=0$ & $\psi^{\prime}=0$ & $\psi^{\prime}=0$ \\
\hline$\left(\mathbf{w}_{b}, 1\right),\left(\frac{a}{2}, 0\right)$ & & $\psi=0$ & $\psi=0$ & $\psi=0$ & $\psi=0$ \\
\hline$\left(\mathbf{w}_{c}, 0\right),\left(\frac{a}{2}, 0\right)$ & & $\psi^{\prime}=0$ & $\psi=0$ & $\psi=0$ & $\psi^{\prime}=0$ \\
\hline$\left(\mathbf{w}_{c}, 1\right),\left(\frac{a}{2}, 0\right)$ & & $\psi=0$ & $\psi^{\prime}=0$ & $\psi^{\prime}=0$ & $\psi=0$ \\
\hline$\left(\mathbf{w}_{d}, 0\right),\left(\frac{a}{2}, 0\right)$ & & $\psi^{\prime}=0$ & $\psi^{\prime}=0$ & $\psi=0$ & $\psi=0$ \\
\hline$\left(\mathbf{w}_{d}, 1\right),\left(\frac{a}{2}, 0\right)$ & & $\psi=0$ & $\psi=0$ & $\psi^{\prime}=0$ & $\psi^{\prime}=0$ \\
\hline$\left(\mathbf{w}_{a}, 0\right),\left(0, \frac{a}{2}\right)$ & & $\psi^{\prime}=0$ & $\psi^{\prime}=0$ & $\psi=0$ & $\psi=0$ \\
\hline$\left(\mathbf{w}_{a}, 1\right),\left(0, \frac{a}{a}\right)$ & & $\psi=0$ & $\psi=0$ & $\psi^{\prime}=0$ & $\psi^{\prime}=0$ \\
\hline$\left(\mathbf{w}_{b}, 0\right),\left(0, \frac{a}{2}\right)$ & & $\psi^{\prime}=0$ & $\psi=0$ & $\psi=0$ & $\psi^{\prime}=0$ \\
\hline$\left(\mathbf{w}_{b}, 1\right),\left(0, \frac{a}{2}\right)$ & & $\psi=0$ & $\psi^{\prime}=0$ & $\psi^{\prime}=0$ & $\psi=0$ \\
\hline$\left(\mathbf{w}_{c}, 0\right),\left(0, \frac{a}{2}\right)$ & & $\psi^{\prime}=0$ & $\psi^{\prime}=0$ & $\psi^{\prime}=0$ & $\psi^{\prime}=0$ \\
\hline$\left(\mathbf{w}_{c}, 1\right),\left(0, \frac{a}{2}\right)$ & & $\psi=0$ & $\psi=0$ & $\psi=0$ & $\psi=0$ \\
\hline$\left(\mathbf{w}_{d}, 0\right),\left(0, \frac{a}{2}\right)$ & & $\psi^{\prime}=0$ & $\psi=0$ & $\psi^{\prime}=0$ & $\psi=0$ \\
\hline$\left(\mathbf{w}_{d}, 1\right),\left(0, \frac{a}{2}\right)$ & & $\psi=0$ & $\psi^{\prime}=0$ & $\psi=0$ & $\psi^{\prime}=0$ \\
\hline$\left(\mathbf{w}_{a}, 0\right),\left(\frac{a}{2}, \frac{a}{2}\right)$ & & $\psi^{\prime}=0$ & $\psi=0$ & $\psi=0$ & $\psi^{\prime}=0$ \\
\hline$\left(\mathbf{w}_{a}, 1\right),\left(\frac{a}{2}, \frac{a}{2}\right)$ & & $\psi=0$ & $\psi^{\prime}=0$ & $\psi^{\prime}=0$ & $\psi=0$ \\
\hline$\left(\mathbf{w}_{b}, 0\right),\left(\frac{a}{2}, \frac{a}{2}\right)$ & & $\psi^{\prime}=0$ & $\psi^{\prime}=0$ & $\psi=0$ & $\psi=0$ \\
\hline$\left(\mathbf{w}_{b}, 1\right),\left(\frac{a}{2}, \frac{a}{2}\right)$ & & $\psi=0$ & $\psi=0$ & $\psi^{\prime}=0$ & $\psi^{\prime}=0$ \\
\hline$\left(\mathbf{w}_{c}, 0\right),\left(\frac{a}{2}, \frac{a}{2}\right)$ & & $\psi^{\prime}=0$ & $\psi=0$ & $\psi^{\prime}=0$ & $\psi=0$ \\
\hline$\left(\mathbf{w}_{c}, 1\right),\left(\frac{a}{2}, \frac{a}{2}\right)$ & & $\psi=0$ & $\psi^{\prime}=0$ & $\psi=0$ & $\psi^{\prime}=0$ \\
\hline$\left(\mathbf{w}_{d}, 0\right),\left(\frac{a}{2}, \frac{a}{2}\right)$ & & $\psi^{\prime}=0$ & $\psi^{\prime}=0$ & $\psi^{\prime}=0$ & $\psi^{\prime}=0$ \\
\hline$\left(\mathbf{w}_{d}, 1\right),\left(\frac{a}{2}, \frac{a}{2}\right)$ & & $\psi=0$ & $\psi=0$ & $\psi=0$ & $\psi=0$ \\
\hline
\end{tabular}


Table 9: The character table and phase factors associated with the point group $C_{3}$, relevant at Wyckoff positions in a $p_{3}$-symmetric atomic insulator.

\begin{tabular}{rrrr}
\hline \hline & $E$ & $C_{3}$ & $C_{3}^{2}$ \\
\hline$D_{0}$ & 1 & 1 & 1 \\
$D_{1}$ & 1 & $e^{i \frac{2 \pi}{3}}$ & $e^{-i \frac{2 \pi}{3}}$ \\
$D_{2}$ & 1 & $e^{-i \frac{2 \pi}{3}}$ & $e^{i \frac{2 \pi}{3}}$ \\
\hline
\end{tabular}

\begin{tabular}{crr}
\hline \hline $\mathbf{w}$ & $\mathbf{R}_{\mathbf{w}}^{C_{3}}$ & $\mathbf{R}_{\mathbf{w}}^{C_{3}^{2}}$ \\
\hline $\mathbf{w}_{a}=(0,0)$ & $\mathbf{0}$ & $\mathbf{0}$ \\
$\mathbf{w}_{b}=\left(\frac{2 a}{3}, \frac{a}{3}\right)$ & $a \hat{\mathbf{e}}_{1}$ & $a\left(\hat{\mathbf{e}}_{1}+\hat{\mathbf{e}}_{2}\right)$ \\
$\mathbf{w}_{c}=\left(\frac{a}{3}, \frac{2 a}{3}\right)$ & $a\left(\hat{\mathbf{e}}_{1}+\hat{\mathbf{e}}_{2}\right)$ & $a \hat{\mathbf{e}}_{2}$ \\
\hline
\end{tabular}

\section{A.3 $p_{3}$ symmetry}

In a $p_{3}$-symmetric crystal, there are three inequivalent Wyckoff positions, at $(0,0),(2 a / 3, a / 3)$, and $(a / 3,2 a / 3)\left[46\right.$, written as $(x, y) \equiv x \hat{\mathbf{e}}_{1}+y \hat{\mathbf{e}}_{2}$ in terms of the unit vectors $\hat{\mathbf{e}}_{1}=$ $-\frac{1}{2} \hat{\mathbf{e}}_{x}+\frac{\sqrt{3}}{2} \hat{\mathbf{e}}_{y}$ and $\hat{\mathbf{e}}_{2}=\hat{\mathbf{e}}_{x}$. These positions are left invariant by the symmetry operations of the point group $C_{3}$, with the character table [25] and phase factors presented in (9). The symmetry operations include $120^{\circ}$ and $240^{\circ}$ rotations, denoted as $C_{3}$ and $C_{3}^{2}$ respectively.

For representations of $p_{3}$-symmetry which do not allow real-valued Wannier functions, the behavior of the logarithmic derivative is less constrained. As noticed before, the effects of the space group symmetry on a crystal edge are most pronounced along edges that respect one of the non-trivial symmetry operations. Since it is not possible for any individual edge to be left invariant under three-fold rotations, we consider a crystal shaped like an equilateral triangle (see figure 3), whose sides are mapped onto one another under $C_{3}$ rotations. The corners are at positions $-N a \hat{\mathbf{e}}_{2}, N a\left(\hat{\mathbf{e}}_{1}+\hat{\mathbf{e}}_{2}\right)$, and $-N a \hat{\mathbf{e}}_{1}$, with $N$ an integer, while the normal vectors to the boundaries are given by $\mathbf{n}_{1}=\hat{\mathbf{e}}_{1}, \mathbf{n}_{2}=\hat{\mathbf{e}}_{2}$ and $\mathbf{n}_{3}=-\left(\hat{\mathbf{e}}_{1}+\hat{\mathbf{e}}_{2}\right)$.

The normal derivatives at the three boundaries can be expressed in terms of just two functions:

$$
\begin{aligned}
& B_{1}^{(\mathbf{w}, l+1)}=\left(e^{i \pi / 3} \hat{\mathbf{e}}_{1}+\hat{\mathbf{e}}_{2}\right) \cdot \nabla \psi^{(\mathbf{w}, l)} \\
& B_{2}^{(\mathbf{w}, l-1)}=\left(e^{-i \pi / 3} \hat{\mathbf{e}}_{1}+\hat{\mathbf{e}}_{2}\right) \cdot \nabla \psi^{(\mathbf{w}, l)} .
\end{aligned}
$$
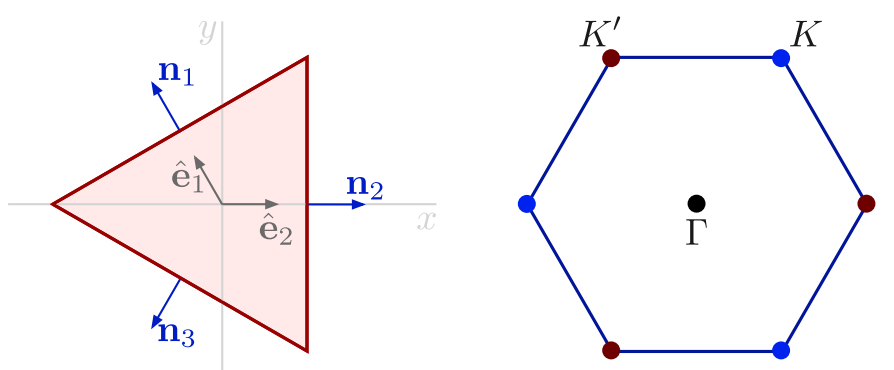

Figure 3: Left: a triangular crystal with three-fold rotational symmetry. The unit vectors $\hat{\mathbf{e}}_{1}$ and $\hat{\mathbf{e}}_{2}$ are indicated, as well as the normal vectors to the boundaries. Right: the corresponding Brillouin zone, with high-symmetry points indicated. 
In terms of these functions, the normal derivatives at the boundaries become:

$$
\begin{aligned}
& \mathbf{n}_{1} \cdot \nabla \psi^{(\mathbf{w}, l)}=\frac{e^{-i \frac{\pi}{2}}}{\sqrt{3}}\left(B_{1}^{(\mathbf{w}, l+1)}-B_{2}^{(\mathbf{w}, l-1)}\right) \\
& \mathbf{n}_{2} \cdot \nabla \psi^{(\mathbf{w}, l)}=\frac{1}{\sqrt{3}}\left(e^{i \pi / 6} B_{1}^{(\mathbf{w}, l+1)}+e^{-i \pi / 6} B_{2}^{(\mathbf{w}, l-1)}\right) \\
& \mathbf{n}_{3} \cdot \nabla \psi^{(\mathbf{w}, l)}=\frac{1}{\sqrt{3}}\left(e^{i 5 \pi / 6} B_{1}^{(\mathbf{w}, l+1)}+e^{-i 5 \pi / 6} B_{2}^{(\mathbf{w}, l-1)}\right) .
\end{aligned}
$$

The indices $l+1$ and $l-1$ of the functions $B_{1}$ and $B_{2}$ indicate that these transform as representations $D_{l \pm 1}$ under the operations of the $C_{3}$ point group. The constraints of equation (3) can therefore also be applied directly to the functions $B_{1}$ and $B_{2}$.

The three-fold rotations leave invariant the Brillouin zone points $\boldsymbol{\Gamma}, \mathbf{K}$, and $\mathbf{K}^{\prime}$. Symmetry-imposed zeroes of the Bloch wave function or its directional derivatives may occur at these points, for real-space locations coinciding with any of the three Wyckoff positions. They are listed in table 10 .

\section{B The logarithmic derivative}

\section{B.1 Symmetry-appropriate derivatives}

Whenever the point group symmetry of a crystal allows for the Wannier functions to be real-valued, the symmetry transformations may not only impose zeroes on the Bloch wave function and its derivatives, but also guarantee the presence of boundary modes [40]. This is most conveniently seen by considering the logarithmic derivative, defined as:

$$
\rho^{(\mathbf{w}, l)}(\mathbf{k}, \mathbf{r})=\frac{\mathbf{n} \cdot \nabla \psi^{(\mathbf{w}, l)}(\mathbf{k}, \mathbf{r})}{\psi^{(\mathbf{w}, l)}(\mathbf{k}, \mathbf{r})} .
$$

To predict whether or not boundary modes are present at any given edge of the crystal, we need to consider the transformation properties of the logarithmic derivative under point group operations. We begin by representing the directional derivatives of the Bloch wave function as:

$$
B^{(\mathbf{w}, \tilde{l})}(\mathbf{k} ; x, y)=\left(\eta_{1} \frac{\partial}{\partial x}+\eta_{2} \frac{\partial}{\partial y}\right) \psi^{(\mathbf{w}, l)}(\mathbf{k} ; x, y) .
$$

Here, the coefficients $\eta_{1}$ and $\eta_{2}$ can be used for example to specify the orientation of the crystal edge.

The functions $B^{(\mathbf{w}, \tilde{l})(\mathbf{k} ; x, y)}$ are called symmetry appropriate functions if, just like the Bloch states, they transform as an irreducible representation of the point group keeping the point $(x, y)$ fixed. The index $\tilde{l}$ then indicates that the function $B^{(\mathbf{w}, \tilde{l})}$ transforms as the representation $D_{\tilde{l}}$. Analogous to equation (3), this implies for a symmetry operation $\alpha$ centered at Wyckoff position $\mathbf{w}$ that:

$$
\alpha B^{(\mathbf{w}, \tilde{l})}(\mathbf{k}, \mathbf{r})=\exp \left(i \mathbf{k} \cdot \mathbf{R}_{\mathbf{w}}^{\alpha}\right) D^{(\tilde{l})}(\alpha) B^{(\mathbf{w}, \tilde{l})}(\mathbf{k}, \mathbf{r})
$$

For the space groups $p_{m m}$ and $p_{2}$, the characters $D^{(\tilde{l})}\left(\alpha_{\mathbf{w}}\right)$ and phase factors $\mathbf{R}_{\mathbf{w}}^{\alpha}$ are listed in tables 1, 2, and 7. The constraint that the functions $B^{(\mathbf{w}, \tilde{l})}$ have to both be symmetryappropriate functions and be related to $\psi^{(\mathbf{w}, l)}$ according to equation (6), yield a one-to-one relation between the indices $\tilde{l}$ of the symmetry-appropriate derivatives, and the indices $l$ of the Bloch wave functions. 
For example, if the normal vector to the boundary of a $p_{m m}$-symmetric crystal is along $\mathbf{e}_{x}$, we can interpret $B^{(\mathbf{w}, \tilde{l})}$ as the normal derivative of the Bloch wave function by taking $\eta_{2}=0$ in equation (6). Considering the mirror operation $\alpha=M_{x}$ then implies that in order for $B^{(\mathbf{w}, \tilde{l})}$ to be a symmetry-appropriate function, we must have $D^{(\tilde{l})}\left(M_{x}\right)=-D^{(l)}\left(M_{x}\right)$. That is, if $\psi^{(\mathbf{w}, l)}$ is even under the operation $(x, y) \rightarrow(-x, y)$, then $B^{(\mathbf{w}, \tilde{l})}$ is odd, and vice versa. In the same way, we can also establish $D^{(\tilde{l})}\left(M_{y}\right)=D^{(l)}\left(M_{y}\right)$ and $D^{(\tilde{l})}\left(C_{2}\right)=$ $-D^{(l)}\left(C_{2}\right)$, and thus we find for this particular termination of a $p_{m m}$ symmetric crystal that $l=0,1,2,3$ implies $\tilde{l}=3,2,1,0$.

In $p_{2}$-symmetric crystals, the connection between Bloch wave functions and their symmetry-appropriate derivatives is even more straightforward. The two-fold rotation affects both the $x$ and $y$ coordinates, so that $D^{(\tilde{l})}\left(C_{2}\right)=-D^{(l)}\left(C_{2}\right)$ and hence $l=0,1 \Leftrightarrow$ $\tilde{l}=1,0$ for any orientation of the boundary.

Table 10: Zeroes of the Bloch wave function $\psi$ and its directional derivatives $\mathbf{n}_{i} \cdot \nabla \psi$, where $\mathbf{n}_{i}$ can be any of three vectors normal to the crystal boundaries. The zeroes are listed for all high-symmetry points in the Brillouin zone, and real space positions $\mathbf{r}=(0,0)$, $\mathbf{r}=(2 a / 3, a / 3)$, and $\mathbf{r}=(a / 3,2 a / 3)$.

\begin{tabular}{|c|c|c|c|}
\hline$(w, l), \mathbf{r}$ & $\mathbf{k}=\boldsymbol{\Gamma}$ & $\bar{K}$ & $\overline{\mathbf{K}^{\prime}}$ \\
\hline$\left(\mathbf{w}_{a}, 0\right),(0,0)$ & $\mathbf{n}_{i} . \nabla \psi=0$ & $\mathbf{n}_{i} . \nabla \psi=0$ & $\mathbf{n}_{i} \cdot \nabla \psi=0$ \\
\hline$\left(\mathbf{w}_{a}, 1\right),(0,0)$ & $\psi=0$ & $\psi=0$ & $\psi=0$ \\
\hline$\left(\mathbf{w}_{a}, 2\right),(0,0)$ & $\psi=0$ & $\psi=0$ & $\psi=0$ \\
\hline$\left(\mathbf{w}_{b}, 0\right),(0,0)$ & $\mathbf{n}_{i} . \nabla \psi=0$ & $\mathbf{n}_{i} \cdot \nabla \psi=0$ & $\mathbf{n}_{i} \cdot \nabla \psi=0$ \\
\hline$\left(\mathbf{w}_{b}, 1\right),(0,0)$ & $\psi=0$ & $\psi=0$ & $\psi=0$ \\
\hline$\left(\mathbf{w}_{b}, 2\right),(0,0)$ & $\psi=0$ & $\psi=0$ & $\psi=0$ \\
\hline$\left(\mathbf{w}_{c}, 0\right),(0,0)$ & $\mathbf{n}_{i} . \nabla \psi=0$ & $\mathbf{n}_{i} \cdot \nabla \psi=0$ & $\mathbf{n}_{i} \cdot \nabla \psi=0$ \\
\hline$\left(\mathbf{w}_{c}, 1\right),(0,0)$ & $\psi=0$ & $\psi=0$ & $\psi=0$ \\
\hline$\left(\mathbf{w}_{c}, 2\right),(0,0)$ & $\psi=0$ & $\psi=0$ & $\psi=0$ \\
\hline$\left(\mathbf{w}_{a}, 0\right),\left(\frac{2 a}{3}, \frac{a}{3}\right)$ & $\mathbf{n}_{i} . \nabla \psi=0$ & $\psi=0$ & $\psi=0$ \\
\hline$\left(\mathbf{w}_{a}, 1\right),\left(\frac{2 a}{3}, \frac{a}{3}\right)$ & $\psi=0$ & $\psi=0$ & $\mathbf{n}_{i} \cdot \nabla \psi=0$ \\
\hline$\left(\mathbf{w}_{a}, 2\right),\left(\frac{2 a}{3}, \frac{a}{3}\right)$ & $\psi=0$ & $\mathbf{n}_{i} \cdot \nabla \psi=0$ & $\psi=0$ \\
\hline$\left(\mathbf{w}_{b}, 0\right),\left(\frac{2 a}{3}, \frac{a}{3}\right)$ & $\mathbf{n}_{i} . \nabla \psi=0$ & $\mathbf{n}_{i} \cdot \nabla \psi=0$ & $\mathbf{n}_{i} \cdot \nabla \psi=0$ \\
\hline$\left(\mathbf{w}_{b}, 1\right),\left(\frac{2 a}{3}, \frac{a}{3}\right)$ & $\psi=0$ & $\psi=0$ & $\psi=0$ \\
\hline$\left(\mathbf{w}_{b}, 2\right),\left(\frac{2 a}{3}, \frac{a}{3}\right)$ & $\psi=0$ & $\psi=0$ & $\psi=0$ \\
\hline$\left(\mathbf{w}_{c}, 0\right),\left(\frac{2 a}{3}, \frac{a}{3}\right)$ & $\mathbf{n}_{i} . \nabla \psi=0$ & $\psi=0$ & $\psi=0$ \\
\hline$\left(\mathbf{w}_{c}, 1\right),\left(\frac{2 a}{3}, \frac{a}{3}\right)$ & $\psi=0$ & $\mathbf{n}_{i} \cdot \nabla \psi=0$ & $\psi=0$ \\
\hline$\left(\mathbf{w}_{c}, 2\right),\left(\frac{2 a}{3}, \frac{a}{3}\right)$ & $\psi=0$ & $\psi=0$ & $\mathbf{n}_{i} \cdot \nabla \psi=0$ \\
\hline$\left(\mathbf{w}_{a}, 0\right),\left(\frac{a}{3}, \frac{2 a}{3}\right)$ & $\mathbf{n}_{i} . \nabla \psi=0$ & $\psi=0$ & $\psi=0$ \\
\hline$\left(\mathbf{w}_{a}, 1\right),\left(\frac{a}{3}, \frac{2 a}{3}\right)$ & $\psi=0$ & $\mathbf{n}_{i} \cdot \nabla \psi=0$ & $\psi=0$ \\
\hline$\left(\mathbf{w}_{a}, 2\right),\left(\frac{a}{3}, \frac{2 a}{3}\right)$ & $\psi=0$ & $\psi=0$ & $\mathbf{n}_{i} \cdot \nabla \psi=0$ \\
\hline$\left(\mathbf{w}_{b}, 0\right),\left(\frac{a}{3}, \frac{2 a}{3}\right)$ & $\mathbf{n}_{i} . \nabla \psi=0$ & $\psi=0$ & $\psi=0$ \\
\hline$\left(\mathbf{w}_{b}, 1\right),\left(\frac{a}{3}, \frac{2 a}{3}\right)$ & $\psi=0$ & $\psi=0$ & $\mathbf{n}_{i} \cdot \nabla \psi=0$ \\
\hline$\left(\mathbf{w}_{b}, 2\right),\left(\frac{a}{3}, \frac{2 a}{3}\right)$ & $\psi=0$ & $\mathbf{n}_{i} \cdot \nabla \psi=0$ & $\psi=0$ \\
\hline$\left(\mathbf{w}_{c}, 0\right),\left(\frac{a}{3}, \frac{2 a}{3}\right)$ & $\mathbf{n}_{i} . \nabla \psi=0$ & $\mathbf{n}_{i} \cdot \nabla \psi=0$ & $\mathbf{n}_{i} \cdot \nabla \psi=0$ \\
\hline$\left(\mathbf{w}_{c}, 1\right),\left(\frac{a}{3}, \frac{2 a}{3}\right)$ & $\psi=0$ & $\psi=0$ & $\psi=0$ \\
\hline$\left(\mathbf{w}_{c}, 2\right),\left(\frac{a}{3}, \frac{2 a}{3}\right)$ & $\psi=0$ & $\psi=0$ & $\psi=0$ \\
\hline
\end{tabular}




\section{B.2 Gauge transformations and lattice translations}

Notice that the logarithmic derivative is invariant under both gauge transformations and translations by any lattice vector. The gauge transformations arise from the fact that Bloch functions for simple bands are defined up to a $\mathbf{k}$ dependent phase factor, and may be written as $\psi^{(\mathbf{w}, l)}(\mathbf{k}, \mathbf{r}) \rightarrow e^{i \phi(\mathbf{k})} \psi^{(\mathbf{w}, l)}(\mathbf{k}, \mathbf{r})$ [26, 47]. Translations by a lattice vector $\mathbf{R}$ similarly affect Bloch functions for simple bands as $\psi^{(\mathbf{w}, l)}(\mathbf{k}, \mathbf{r}) \rightarrow e^{i \mathbf{k} \cdot \mathbf{R}} \psi^{(\mathbf{w}, l)}(\mathbf{k}, \mathbf{r})$. Since neither of the phase factors used in these transformations depends on the spatial coordinate $\mathbf{r}$, they can be taken outside the gradient in equation (5), and leave the logarithmic derivative invariant.

\section{B.3 Real-valued Wannier functions}

For real-valued Wannier functions, the logarithmic derivative behaves under complex conjugation as [40]:

$$
\rho^{*}(\mathbf{k}, \mathbf{r})=\rho\left(-\mathbf{k}_{R}+i \mathbf{k}_{I}, \mathbf{r}\right), \quad \text { with } \mathbf{k}=\mathbf{k}_{R}+i \mathbf{k}_{I} .
$$

Solutions of Schrödinger's equation with complex $\mathbf{k}$ here represent boundary modes that decay exponentially in real space.

Both $p_{m m}$ and $p_{2}$ allow real-valued Wannier functions. They share the same four highsymmetry points in reciprocal space, all of which have the property that $\mathbf{k}_{R}=-\mathbf{k}_{R}$ up to translations by a reciprocal lattice vector. From equation (7) it is then clear that the logarithmic derivative at these points is a real-valued function. This includes in particular any boundary modes that arise at high symmetry values of $\mathbf{k}_{R}$.

Combining the transformation of the logarithmic derivative under complex conjugation with its transformation properties under symmetry group operations restricts the logarithmic derivative even further. Since both the Bloch wave functions $\psi^{(\mathbf{w}, l)}$ and their derivatives $B^{(\mathbf{w}, \tilde{l})}$ are constructed from Wannier functions centered at the Wyckoff position $\mathbf{w}$, they share the same associated phase factors $e^{i \mathbf{k} \cdot \mathbf{R}_{\mathbf{w}}^{\alpha}}$ for point group operations centered at $\mathbf{w}$ [31]. Applying equation (3) to the logarithmic derivative then yields:

$$
\rho\left(\alpha^{-1} \mathbf{k}, \alpha^{-1} \mathbf{r}\right)=\frac{D^{(\tilde{l})}(\alpha)}{D^{(l)}(\alpha)} \rho(\mathbf{k}, \mathbf{r})
$$

For the special case in which the momentum $\mathbf{k}$ is purely real and the real-space position $\mathbf{r}$ is a Wyckoff position, this relation reduces to:

$$
\rho\left(\alpha^{-1} \mathbf{k}_{R}, \mathbf{w}\right)=\frac{D^{(\tilde{l})}(\alpha)}{D^{(l)}(\alpha)} \rho\left(\mathbf{k}_{R}, \mathbf{w}\right) .
$$

Considering for example a $p_{2}$-symmetric crystal, and taking $\alpha$ to be the two-fold rotation yields $\alpha^{-1} \mathbf{k}_{R}=-\mathbf{k}_{R}$ and $D^{(\tilde{l})}(\alpha) / D^{(l)}(\alpha)=-1$. Combining equations (7) and (9) then leads to the constraint that the logarithmic derivative must be purely imaginary for any real $\mathbf{k}$. The same argument applies to any crystal with inversion symmetry, while considering only a single mirror operation (say $M_{x}$ ) suffices to render the logarithmic derivative purely imaginary only for real momenta along lines in the Brillouin zone $\left(k_{y}=0\right.$ and $k_{y}=\pi$ ) and for specific orientations of the crystal boundary (parallel to $\hat{\mathbf{e}}_{y}$ ).

As explained in the main text, the constraints discussed above for crystals whose space group includes the inversion operation and allows for real-valued Wannier functions [48], suffice to determine whether or not the logarithmic derivative changes sign from one highsymmetry momentum value to another. This in turn determines whether or not any boundary states arise at the high-symmetry momenta. 


\section{B.4 The sign of the logarithmic derivative}

To predict the presence of interface states at junction interfaces, we first establish how the signs of the logarithmic derivative for in-gap states depends on its zeroes and divergences at high-symmetry points. For concreteness, we consider momentum values going from $\boldsymbol{\Gamma}=(0,0)$ to $\mathbf{X}=(\pi / a, 0)$, but the same reasoning can be applied to any other pair of high symmetry points. Whether $\rho=0$ or $\rho=\infty$ for $\mathbf{k}$ and $\mathbf{r}$ fixed at high symmetry positions (in the Brillouin zone and real space respectively), can be deduced from tables 3 to 8 ,

First, consider the case of $\rho(\Gamma, \mathbf{r})=0$, with $\mathbf{r}$ a high-symmetry real-space position at the boundary of the crystal. For the logarithmic derivative to vanish, we must have that $B^{(\mathbf{w}, \tilde{l})}(\mathbf{k}, \mathbf{r})=B^{(\mathbf{w}, \tilde{l})}\left(\left[k_{x}, k_{y}\right], \mathbf{r}\right)$ vanishes as well. We can then use the analytic nature of $B^{(\mathbf{w}, \tilde{l})}$ to write it as a power series for momenta close to $\boldsymbol{\Gamma}$ and approximate it as:

$$
\begin{aligned}
B^{(\mathbf{w}, \tilde{l})}\left(\left[k_{x}, 0\right], \mathbf{r}\right) & =\sum_{n} c_{n} k_{x}^{n} \\
\Rightarrow B^{(\mathbf{w}, \tilde{l})}([ \pm i \delta, 0], \mathbf{r}) & \sim \delta \quad \text { for } \delta<<1 .
\end{aligned}
$$

Here, we used the fact that for in-gap states, the logarithmic derivative is real, and we assumed it to be positive in this example, without loss of generality. Using continuity, it immediately follows that $B^{(\mathbf{w}, \tilde{l})}([\delta, 0], \mathbf{r}) \sim \mp i \delta$.

Given that the logarithmic derivative for real momenta is purely imaginary, $\rho$ remains constrained to the imaginary axis as we transverse the band, and can only change sign when either $B^{(\mathbf{w}, \tilde{l})}$ or $\psi^{(\mathbf{w}, l)}$ goes to zero. This is forced by symmetry to happen again only at $\mathbf{k}=\mathbf{X}$, where we can again introduce a power series expansion:

$$
B^{(\mathbf{w}, \tilde{l})}\left(\left[k_{x}, 0\right], \mathbf{r}\right)=\sum_{n} d_{n}\left(k_{x}-\pi / a\right)^{n} .
$$

Considering the case in which the logarithmic derivative vanishes at $\mathbf{X}$, and using the fact that $\rho$ could not change sign while traversing the band from $\boldsymbol{\Gamma}$ to $\mathbf{X}$, then yields:

$$
\begin{aligned}
B^{(\mathbf{w}, \tilde{l})}([\pi / a-\delta, 0], \mathbf{r}) & \sim \mp i \delta \\
B^{(\mathbf{w}, \tilde{l})}([\pi / a \pm i \delta, 0], \mathbf{r}) & \sim-\delta \quad \text { for } \delta<<1,
\end{aligned}
$$

where in the final line, we again used continuity of $B^{(\mathbf{w}, \tilde{l})}$. The logarithmic derivatives in the gaps bordering $\mathbf{\Gamma}$ and $\mathbf{X}$ thus have opposite sign, and more generally, the sign of $\rho$ for in-gap states changes from one gap to the next if the gaps are connected by a band with vanishing logarithmic derivatives at both high-symmetry momenta bordering the gaps. Note that for a given position of the boundary, tables 3 to 8 can be used to identify the band labels $(\mathbf{w}, l)$ that yield zeroes at any pair of high symmetry momenta.

Next, consider the case of the logarithmic derivative diverging at both high symmetry momenta. The same type of series expansions can now be made for the wave function $\psi^{(\mathbf{w}, l)}$ rather than the symmetry-appropriate derivative. Close to $\boldsymbol{\Gamma}$, we can then start from $\psi^{(\mathbf{w}, l)}([ \pm i \delta, 0], \mathbf{r}) \sim \delta \Rightarrow \rho([\delta, 0], \mathbf{r}) \sim \pm i / \delta$. Following the same reasoning as before, this in turn implies $\rho([\pi / a-\delta, 0], \mathbf{r}) \sim \pm i / \delta$, and hence $\rho([\pi / a \pm \delta, 0], \mathbf{r}) \sim-1 / \delta$. Thus, the logarithmic derivative has opposite signs in two gaps connected by a band with equal behaviour of the logarithmic derivative at its two endpoints, regardless of whether it goes to zero or diverges.

The final case to consider has a vanishing logarithmic derivative at one high symmetry momentum, and a divergence at the other. For concreteness we take $\boldsymbol{\Gamma}$ to have $\rho=0$ while $\rho=\infty$ at $\mathbf{X}$, but the result is general. We then start with $\rho([ \pm i \delta, 0], \mathbf{r}) \sim \delta$, 


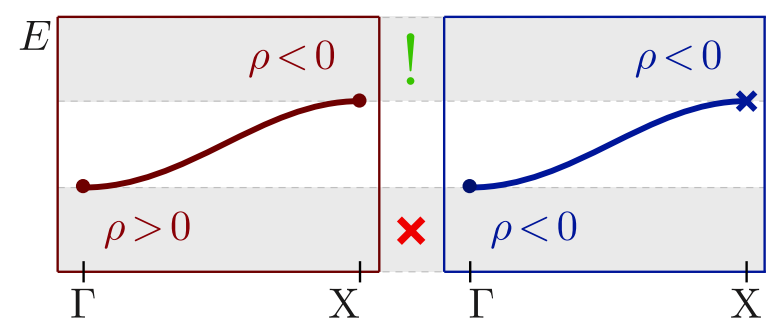

Figure 4: An examples of energy bands of neighbouring crystalline insulators in a heterojunction architecture. The indicated signs of the logarithmic derivatives $\rho$ for in-gap states are uniquely determine the zeroes of the Bloch wave function (solid dots) and its derivative (crosses) at high-symmetry points in the Brillouin zone. A topological obstruction for bulk states to connect across the interface arises when the signs of the logarithmic derivatives within a particular gap agree across the junction, as for the top gap. A localised topological state then emerges at the interface.

leading to $\rho([\delta, 0], \mathbf{r}) \sim \mp i \delta$. This fixes the sign of the purely imaginary logarithmic derivative as we traverse the band from $\boldsymbol{\Gamma}$ to $\mathbf{X}$. Close to $\mathbf{X}$, where $\rho$ diverges, we then find $\rho([\pi / a-\delta, 0], \mathbf{r}) \sim \mp i / \delta$. Continuity finally allows us to infer that $\rho([\pi / a \pm i \delta, 0], \mathbf{r}) \sim 1 / \delta$, and hence that the logarithmic derivative for in-gap states close to $\mathbf{X}$ has the same sign as for in-gap states close to $\boldsymbol{\Gamma}$. More generally, we thus find that there are two possible scenarios: if a gap at one high symmetry momentum is connected to a gap at another high symmetry point by a band whose logarithmic derivative has the same behaviour at both endpoints, the signs of $\rho$ for the in-gap states are opposite, while bands with different behavior of the logarithmic derivative at the high symmetry points yield equal signs for the in-gap states.

\section{B.5 Independence from boundary shifts in $p_{2}$ and $p_{m m}$}

Whether the signs of logarithmic derivatives match across an interface separating two atomic insulators, depends on the location of the interface as well as on the band structures of the two insulators. Nevertheless, the prediction of whether or not interface states exist in junctions between two atomic insulators with the same space group, is robust to spatial shifts (or distortions) of the interface from one Wyckoff position to any other. To see this, consider one band each in two insulators joined together in a heterojunction architecture (as shown in Fig. 4). The bands in the two insulators in the top panel have different limiting behaviours for their logarithmic derivatives. The material on the left has vanishing $\rho$ at both $\boldsymbol{\Gamma}$ and $\mathbf{X}$, while the material on the right has one zero and one divergence.

The zeroes of the Bloch function $\psi^{(\mathbf{w}, l)}$ and its symmetry-appropriate derivative $B^{(\mathbf{w}, \tilde{l})}$ are determined by their symmetry transformations, which read:

$$
\begin{aligned}
& \left(1-e^{i \mathbf{k} \cdot\left(\mathbf{R}_{\mathbf{w}}^{\alpha}-\mathbf{R}\right)} D^{(l)}(\alpha)\right) \psi^{(\mathbf{w}, l)}(\mathbf{k}, \mathbf{r})=0 \\
& \left(1-e^{i \mathbf{k} \cdot\left(\mathbf{R}_{\mathbf{w}}^{\alpha}-\mathbf{R}\right)} D^{(\tilde{l})}(\alpha)\right) B^{(\mathbf{w}, \tilde{l})}(\mathbf{k}, \mathbf{r})=0 .
\end{aligned}
$$

Here $\mathbf{R}$ is a reciprocal lattice vector, defined through the relation $\alpha^{-1} \mathbf{r}=\mathbf{r}+\mathbf{R}$. In $p 2$ and $p m m$-symmetric crystals, the symmetry operations require either the Bloch function or its derivative to go to zero at high symmetry momenta. We must therefore either have $e^{i \mathbf{k} \cdot\left(\mathbf{R}_{\mathbf{w}}^{\alpha}-\mathbf{R}\right)} D^{(l)}(\alpha)=1$, or $e^{i \mathbf{k} \cdot\left(\mathbf{R}_{\mathbf{w}}^{\alpha}-\mathbf{R}\right)} D^{(\tilde{l})}(\alpha)=1$ at those positions in the Brillouin zone. 
If we now change $\mathbf{r}$ from one high symmetry position in real space to another, the only effect this can have in Eq. (13), is to change the value of $\mathbf{R}$ in the phase factor. This change upon moving the location of the interface from $\mathbf{r}$ to $\mathbf{r}^{\prime}=\mathbf{r}+\delta \mathbf{r}$ is determined by action of the symmetry operations: $\mathbf{R}^{\prime}=\alpha^{-1} \mathbf{r}^{\prime}-\mathbf{r}^{\prime}=\mathbf{R}+\alpha^{-1} \delta \mathbf{r}-\delta \mathbf{r}$. The two crystals on opposite sides of the junction thus both acquire an additional term $\mathbf{k} \cdot\left(\alpha^{-1} \delta \mathbf{r}-\delta \mathbf{r}\right)$ in their phase factors. For a given high symmetry momentum value, if for example the condition $e^{i \mathbf{k} \cdot\left(\mathbf{R}_{\mathbf{w}}^{\alpha}-\mathbf{R}\right)} D^{(l)}(\alpha)=1$ was satisfied with the interface at $\mathbf{r}$, the additional phase factor associated with moving the interface to $\mathbf{r}^{\prime}$ can either cause the condition to remain satisfied, or to be violated. Since there must always be a zero of either the Bloch function or its derivative, the latter case would cause the condition $e^{i \mathbf{k} \cdot\left(\mathbf{R}_{\mathbf{w}}^{\alpha}-\mathbf{R}^{\prime}\right)} D^{(\tilde{l})}(\alpha)=1$ on the representation of the derivative to become satisfied. But since the crystal on the other side of the junction incurs the same additions to its phase factors, it must undergo the same pattern of either keeping its zero at $\mathbf{k}$ fixed or shifting it between the Bloch function and its derivative.

This argument holds for all high symmetry momenta, and thus guarantees that at any given point in the Brillouin zone either the zeroes and divergences on both sides of the junction are unaltered by a shift of the interface, or they change simultaneously. Either way, whether or not the signs of the in-gap logarithmic derivatives change from the gap below the band to the gap above it, is affected in the same way on either side of the junction. A gap with matching signs for an interface at $\mathbf{r}$ will therefore also have matching signs with the interface at $\mathbf{r}^{\prime}$, and similarly for gaps in which the signs do not match. Changing the location of the interface, from one high symmetry location in real space to another therefore does not affect the existence of interface states for junctions in which the crystals are either both $p 2$ or both $p m m$-symmetric.

\section{Momentum-space irreducible representations}

For atomic insulators it is always possible to induce the momentum space representations at high-symmetry points from the real-space band labels 32,45 . Notice however that the former are more general, in the sense that they describe strong topological insulators with non-zero Chern numbers as well as atomic insulators, whereas the real-space band symmetry labels can be applied only to atomic insulators $19,30,39$. For crystals with $p 2$ or $\mathrm{pmm}$ symmetry, we can straightforwardly construct the explicit map from real space to momentum space labels.

\section{C.1 $p_{2}$-symmetric atomic insulators}

For $p_{2}$-symmetric atomic insulators, the irreducible representations of Bloch states at high symmetry points in the Brillouin zone can be deduced directly from the transformation properties associated with the real-space symmetry labels. As an example, consider the wave function $\psi^{\left(\mathbf{w}_{\mathbf{a}}, l\right)}(\mathbf{k}, \mathbf{r})$. The only non-trivial symmetry operation that we can consider for $p 2$-symmetric crystals is the two-fold rotation $C_{2}$. From Eq. (3), we can see that at for example $\mathbf{k}=\boldsymbol{\Gamma}$, this symmetry operation acts on the wave function as:

$$
\begin{aligned}
C_{2} \psi^{\left(\mathbf{w}_{\mathbf{a}}, l\right)}(\boldsymbol{\Gamma}, \mathbf{r}) & =D_{l}\left(C_{2}\right) \psi^{\left(\mathbf{w}_{\mathbf{a}}, l\right)}(\boldsymbol{\Gamma}, \mathbf{r}) \\
& =(-1)^{l} \psi^{\left(\mathbf{w}_{\mathbf{a}}, l\right)}(\boldsymbol{\Gamma}, \mathbf{r}) .
\end{aligned}
$$

At the momentum point $\boldsymbol{\Gamma}$, the wave function is thus even under inversion for the realspace symmetry label with $l=0$, and odd for $l=1$. Consequently, a band with real-space symmetry label $\left(\mathbf{w}_{\mathbf{a}}, 0\right)$ gives rise to the irreducible representation $\Gamma_{0}$ (with character 
Table 11: Correspondence between real-space symmetry labels and momentum-space irreducible representations in a $p 2$-symmetric atomic insulator.

\begin{tabular}{ccccc}
\hline \hline$(w, l)$ & $\boldsymbol{\Gamma}$ & $\mathbf{X}$ & $\mathbf{Y}$ & $\mathbf{M}$ \\
\hline$\left(\mathbf{w}_{a}, 0\right)$ & $\Gamma_{0}$ & $X_{0}$ & $Y_{0}$ & $M_{0}$ \\
$\left(\mathbf{w}_{a}, 1\right)$ & $\Gamma_{1}$ & $X_{1}$ & $Y_{1}$ & $M_{1}$ \\
$\left(\mathbf{w}_{b}, 0\right)$ & $\Gamma_{0}$ & $X_{1}$ & $Y_{0}$ & $M_{1}$ \\
$\left(\mathbf{w}_{b}, 1\right)$ & $\Gamma_{1}$ & $X_{0}$ & $Y_{1}$ & $M_{0}$ \\
$\left(\mathbf{w}_{c}, 0\right)$ & $\Gamma_{0}$ & $X_{0}$ & $Y_{1}$ & $M_{1}$ \\
$\left(\mathbf{w}_{c}, 1\right)$ & $\Gamma_{1}$ & $X_{1}$ & $Y_{0}$ & $M_{0}$ \\
$\left(\mathbf{w}_{d}, 0\right)$ & $\Gamma_{0}$ & $X_{1}$ & $Y_{1}$ & $M_{0}$ \\
$\left(\mathbf{w}_{d}, 1\right)$ & $\Gamma_{1}$ & $X_{0}$ & $Y_{0}$ & $M_{1}$ \\
\hline
\end{tabular}

$+1)$ at the momentum point $\Gamma$, while $\left(\mathbf{w}_{\mathbf{a}}, 1\right)$ generates to the irreducible representation $\Gamma_{1}$ (with character -1 ). Proceeding in this way for the other high symmetry points in the Brillouin zone, we can compute what is the string of irreducible representations in momentum space produced by all the different $(\mathbf{w}, l)$ elementary band representations. The results are illustrated in Table 11, which is consistent with [30].

Notice that Table 11 contains only half of all possible $2^{4}=16$ combinations of even and odd irreducible representations at the four high symmetry points in the Brillouin zone. The combinations in the table are the only ones consistent with Wannier representations indexed by real space symmetry labels $(\mathbf{w}, l)$, and thus the only ones relevant for atomic insulators. The missing combinations of momentum-space irreducible representations represent bands with a non-zero Chern number, which cannot be written in terms of globally defined localised Wannier functions. Here, we focus on the atomic insulators, with zero Chern number.

\section{C.2 $p_{m m}$-symmetric atomic insulators}

Crystals with $p_{m m}$ symmetry possess high symmetry lines as well as high symmetry points. They are given by $l_{1}=\left(k_{x}, 0\right), l_{2}=\left(\pi / a, k_{y}\right), l_{3}=\left(k_{x}, \pi / a\right)$, and $l_{4}=\left(0, k_{y}\right)$, and are left invariant by a subgroup of the full point group: $l_{1}$ and $l_{3}$ are unchanged under the operations $\left\{E, M_{y}\right\}$, while $l_{2}$ and $l_{4}$ are invariant under the action of $\left\{E, M_{x}\right\}$. Each set constitutes a group of its own, which is isomorphic to the cyclic group $Z_{2}$. The representations can be labelled "+" or " - ", depending on whether they are even or odd under the relevant mirror operation.

We can now again use Eq. (3) to deduce the momentum space irreducible representations of both the high symmetry points and the high symmetry lines in the Brillouin zone from the action of the symmetry operations on Bloch functions with any given real-space symmetry label. Consider, for exanple, the elementary band representation $\left(\mathbf{w}_{b}, 0\right)$, which transforms as:

$$
\begin{aligned}
M_{x} \psi^{\left(\mathbf{w}_{b}, 0\right)}(\boldsymbol{\Gamma}, \mathbf{r}) & =M_{y} \psi^{\left(\mathbf{w}_{b}, 0\right)}(\boldsymbol{\Gamma}, \mathbf{r})=\psi^{\left(\mathbf{w}_{b}, 0\right)}(\boldsymbol{\Gamma}, \mathbf{r}) \\
-M_{x} \psi^{\left(\mathbf{w}_{b}, 0\right)}(\mathbf{X}, \mathbf{r}) & =M_{y} \psi^{\left(\mathbf{w}_{b}, 0\right)}(\mathbf{X}, \mathbf{r})=\psi^{\left(\mathbf{w}_{b}, 0\right)}(\mathbf{X}, \mathbf{r}) \\
M_{x} \psi^{\left(\mathbf{w}_{b}, 0\right)}(\mathbf{Y}, \mathbf{r}) & =M_{y} \psi^{\left(\mathbf{w}_{b}, 0\right)}(\mathbf{Y}, \mathbf{r})=\psi^{\left(\mathbf{w}_{b}, 0\right)}(\mathbf{Y}, \mathbf{r}) \\
-M_{x} \psi^{\left(\mathbf{w}_{b}, 0\right)}(\mathbf{M}, \mathbf{r}) & =M_{y} \psi^{\left(\mathbf{w}_{b}, 0\right)}(\mathbf{M}, \mathbf{r})=\psi^{\left(\mathbf{w}_{b}, 0\right)}(\mathbf{M}, \mathbf{r}) .
\end{aligned}
$$


Table 12: Correspondence between real-space symmetry labels and momentum-space irreducible representations in a $p m m$-symmetric atomic insulator. The entry $\left\{l_{1}, l_{2}, l_{3}, l_{4}\right\}$ lists the induced irreducible representations of the high symmetry lines.

\begin{tabular}{cccccc}
\hline \hline$(w, l)$ & $\left\{l_{1}, l_{2}, l_{3}, l_{4}\right\}$ & $\boldsymbol{\Gamma}$ & $\mathbf{X}$ & $\mathbf{Y}$ & $\mathbf{M}$ \\
\hline$\left(\mathbf{w}_{a}, 0\right)$ & $\{+,+,+,+\}$ & $\Gamma_{0}$ & $X_{0}$ & $Y_{0}$ & $M_{0}$ \\
$\left(\mathbf{w}_{a}, 1\right)$ & $\{-,-,-,-\}$ & $\Gamma_{1}$ & $X_{1}$ & $Y_{1}$ & $M_{1}$ \\
$\left(\mathbf{w}_{a}, 2\right)$ & $\{-,+,-,+\}$ & $\Gamma_{2}$ & $X_{2}$ & $Y_{2}$ & $M_{2}$ \\
$\left(\mathbf{w}_{a}, 3\right)$ & $\{+,-,+,-\}$ & $\Gamma_{3}$ & $X_{3}$ & $Y_{3}$ & $M_{3}$ \\
$\left(\mathbf{w}_{b}, 0\right)$ & $\{+,-,+,+\}$ & $\Gamma_{0}$ & $X_{3}$ & $Y_{0}$ & $M_{3}$ \\
$\left(\mathbf{w}_{b}, 1\right)$ & $\{-,+,-,-\}$ & $\Gamma_{1}$ & $X_{2}$ & $Y_{1}$ & $M_{2}$ \\
$\left(\mathbf{w}_{b}, 2\right)$ & $\{-,-,-,+\}$ & $\Gamma_{2}$ & $X_{1}$ & $Y_{2}$ & $M_{1}$ \\
$\left(\mathbf{w}_{b}, 3\right)$ & $\{+,+,+,-\}$ & $\Gamma_{3}$ & $X_{0}$ & $Y_{3}$ & $M_{0}$ \\
$\left(\mathbf{w}_{c}, 0\right)$ & $\{+,+,-,+\}$ & $\Gamma_{0}$ & $X_{0}$ & $Y_{2}$ & $M_{2}$ \\
$\left(\mathbf{w}_{c}, 1\right)$ & $\{-,-,+,-\}$ & $\Gamma_{1}$ & $X_{1}$ & $Y_{3}$ & $M_{3}$ \\
$\left(\mathbf{w}_{c}, 2\right)$ & $\{-,+,+,+\}$ & $\Gamma_{2}$ & $X_{2}$ & $Y_{0}$ & $M_{0}$ \\
$\left(\mathbf{w}_{c}, 3\right)$ & $\{+,-,-,-\}$ & $\Gamma_{3}$ & $X_{3}$ & $Y_{1}$ & $M_{1}$ \\
$\left(\mathbf{w}_{d}, 0\right)$ & $\{+,-,-,+\}$ & $\Gamma_{0}$ & $X_{3}$ & $Y_{2}$ & $M_{1}$ \\
$\left(\mathbf{w}_{d}, 1\right)$ & $\{-,+,+,-\}$ & $\Gamma_{1}$ & $X_{2}$ & $Y_{3}$ & $M_{0}$ \\
$\left(\mathbf{w}_{d}, 2\right)$ & $\{-,-,+,+\}$ & $\Gamma_{2}$ & $X_{1}$ & $Y_{0}$ & $M_{3}$ \\
$\left(\mathbf{w}_{d}, 3\right)$ & $\{+,+,-,-\}$ & $\Gamma_{3}$ & $X_{0}$ & $Y_{1}$ & $M_{2}$ \\
\hline
\end{tabular}

Since the $C_{2}$ element of the point group is simply the product of the two mirror operations, the mirror eigenvalues by themselves already determine the irreducible representations at the high symmetry points. For example, the fact that $\psi^{\left(\mathbf{w}_{b}, 0\right)}(\boldsymbol{\Gamma}, \mathbf{r})$ is even under both mirror operations implies that its irreducible representation at $\boldsymbol{\Gamma}$ is $\Gamma_{0}$. Similarly, the irreducible representations at $\mathbf{X}, \mathbf{Y}$, and $\mathbf{M}$ are found to be $X_{3}, Y_{0}$, and $M_{3}$ respectively.

The irreducible representations associated with the high symmetry lines are induced by those at the high symmetry points. For example, $l_{1}$ is the line connecting $\boldsymbol{\Gamma}$ and $\mathbf{X}$. Since it is mapped onto itself by the operation $M_{y}$, all Bloch states with momenta on the line transform as irreducible representations of the $Z_{2}$ group $\left\{E, M_{y}\right\}$. Since the momentum can be adiabatically changed along the line, however, there can be no discontinuous changes of the irreducible representation along the line, and all states on $l_{1}$ must transform under $M_{y}$ in the same way. In particular, this includes the end points, $\boldsymbol{\Gamma}$ and $\mathbf{X}$. Since both of these transform with eigenvalue +1 under $M_{y}$, all states along $l_{1}$ must also be even under the mirror operation. Continuing this way, Table 12 can be constructed, which lists the irreducible representations associated with all high symmetry lines and points in momentum space for each of the possible elementary band representations associated with a $p m m$-symmetric atomic insulator. 


\section{References}

[1] J. E. Moore, The birth of topological insulators, Nature 464(7286), 194 (2010).

[2] M. Z. Hasan and C. L. Kane, Colloquium: Topological insulators, Rev. Mod. Phys. 82, 3045 (2010), doi:10.1103/RevModPhys.82.3045.

[3] X.-L. Qi and S.-C. Zhang, Topological insulators and superconductors, Reviews of Modern Physics 83(4), 1057 (2011).

[4] W. Han, Y. Otani and S. Maekawa, Quantum materials for spin and charge conversion, npj Quantum Materials 3(1), 1 (2018).

[5] L. Šmejkal, Y. Mokrousov, B. Yan and A. H. MacDonald, Topological antiferromagnetic spintronics, Nature Physics 14(3), 242 (2018).

[6] B. Yan and C. Felser, Topological materials: Weyl semimetals, Annual Review of Condensed Matter Physics 8, 337 (2017).

[7] N. Cooper, J. Dalibard and I. Spielman, Topological bands for ultracold atoms, Reviews of modern physics 91(1), 015005 (2019).

[8] K. Sone and Y. Ashida, Anomalous topological active matter, Physical Review Letters 123(20), 205502 (2019).

[9] Z. Yang, F. Gao, X. Shi, X. Lin, Z. Gao, Y. Chong and B. Zhang, Topological acoustics, Physical review letters 114(11), 114301 (2015).

[10] S. D. Huber, Topological mechanics, Nature Physics 12(7), 621 (2016).

[11] J.-W. Rhim, J. H. Bardarson and R.-J. Slager, Unified bulk-boundary correspondence for band insulators, Physical Review B 97(11), 115143 (2018).

[12] D. J. Thouless, M. Kohmoto, M. P. Nightingale and M. den Nijs, Quantized hall conductance in a two-dimensional periodic potential, Phys. Rev. Lett. 49, 405 (1982), doi:10.1103/PhysRevLett.49.405.

[13] B. I. Halperin, Quantized hall conductance, current-carrying edge states, and the existence of extended states in a two-dimensional disordered potential, Phys. Rev. B 25, 2185 (1982), doi:10.1103/PhysRevB.25.2185.

[14] Y. Hatsugai, Chern number and edge states in the integer quantum hall effect, Phys. Rev. Lett. 71, 3697 (1993), doi:10.1103/PhysRevLett.71.3697.

[15] C. L. Kane and E. J. Mele, $Z_{2}$ topological order and the quantum spin hall effect, Phys. Rev. Lett. 95, 146802 (2005), doi:10.1103/PhysRevLett.95.146802.

[16] L. Fu and C. L. Kane, Topological insulators with inversion symmetry, Phys. Rev. B 76, 045302 (2007), doi:10.1103/PhysRevB.76.045302.

[17] L. Fu, Topological crystalline insulators, Phys. Rev. Lett. 106, 106802 (2011), doi:10.1103/PhysRevLett.106.106802.

[18] J. Kruthoff, J. de Boer, J. van Wezel, C. L. Kane and R.-J. Slager, Topological classification of crystalline insulators through band structure combinatorics, Phys. Rev. X 7, 041069 (2017), doi:10.1103/PhysRevX.7.041069. 
[19] B. Bradlyn, L. Elcoro, J. Cano, M. G. Vergniory, Z. Wang, C. Felser, M. I. Aroyo and B. A. Bernevig, Topological quantum chemistry, Nature 547, 298 (2017), doi:10.1038/nature23268.

[20] H. C. Po, A. Vishwanath and H. Watanabe, Symmetry-based indicators of band topology in the 230 space groups, Nature Communications 8, 50 (2017), doi:10.1038/s41467-017-00133-2.

[21] J. Zak, Berry's phase for energy bands in solids, Phys. Rev. Lett. 62, 2747 (1989), doi:10.1103/PhysRevLett.62.2747.

[22] K. S. Novoselov, A. Mishchenko, A. Carvalho and A. H. Castro Neto, 2d materials and van der waals heterostructures, Science 353, aac9439 (2016), doi:10.1126/science.aac9439.

[23] D. Jariwala, T. J. Marks and M. C. Hersam, Mixed-dimensional van der waals heterostructures, Nature Materials 16, 170 (2017), doi:10.1038/nmat4703.

[24] Y. Liu, N. O. Weiss, X. Duan, H.-C. Cheng, Y. Huang and X. Duan, Van der waals heterostructures and devices, Nat. Rev. Mater. 1, 16042 (2016), doi: $10.1038 /$ natrevmats.2016.42.

[25] M. Tinkham, Group theory and quantum mechanics, Courier Corporation (2003).

[26] W. Kohn, Construction of wannier functions and applications to energy bands, Phys. Rev. B 7, 4388 (1973), doi:10.1103/PhysRevB.7.4388.

[27] L. Michel and J. Zak, Connectivity of energy bands in crystals, Phys. Rev. B 59, 5998 (1999), doi:10.1103/PhysRevB.59.5998.

[28] L. Michel and J. Zak, Elementary energy bands in crystals are connected, Physics Reports 341(1), 377 (2001), doi:https://doi.org/10.1016/S0370-1573(00)00093-4.

[29] J. Cano, B. Bradlyn, Z. Wang, L. Elcoro, M. G. Vergniory, C. Felser, M. I. Aroyo and B. A. Bernevig, Building blocks of topological quantum chemistry: Elementary band representations, Phys. Rev. B 97, 035139 (2018), doi:10.1103/PhysRevB.97.035139.

[30] J. Zak, Do energy bands in solids have an identity, In Group Theoretical Methods in Physics, pp. 463-472. Springer (1984).

[31] J. Zak, Band representations and symmetry types of bands in solids, Phys. Rev. B 23, 2824 (1981), doi:10.1103/PhysRevB.23.2824.

[32] J. Zak, Band representations of space groups, Phys. Rev. B 26, 3010 (1982), doi:10.1103/PhysRevB.26.3010.

[33] H. Bacry, L. Michel and J. Zak, Symmetry and analyticity of energy bands in solids, Phys. Rev. Lett. 61, 1005 (1988), doi:10.1103/PhysRevLett.61.1005.

[34] W. Shockley, On the surface states associated with a periodic potential, Phys. Rev. 56, 317 (1939), doi:10.1103/PhysRev.56.317.

[35] A.-W. Maue, Die oberflächenwellen in der elektronentheorie der metalle, Zeitschrift für Physik 94(11-12), 717 (1935). 
[36] E. T. Goodwin, Electronic states at the surfaces of crystals: I. the approximation of nearly free electrons, Mathematical Proceedings of the Cambridge Philosophical Society 35, 205 (1939), doi:10.1017/S0305004100020910.

[37] A. Silva, Crystal Symmetries in Charge-Orbital Order and Topological Band Theory, Ph.D. thesis, University of Amsterdam (2019).

[38] G. Panati, Triviality of bloch and bloch-dirac bundles, In Annales Henri Poincaré, vol. 8, pp. 995-1011. Springer (2007).

[39] C. Brouder, G. Panati, M. Calandra, C. Mourougane and N. Marzari, Exponential localization of wannier functions in insulators, Physical review letters 98(4), 046402 (2007).

[40] J. Zak, Symmetry criterion for surface states in solids, Phys. Rev. B 32, 2218 (1985), doi:10.1103/PhysRevB.32.2218.

[41] I. Tamm, Über eine mögliche art der elektronenbindung an kristalloberflächen, In Selected Papers, pp. 91-102. Springer (1991).

[42] W. Kohn, Analytic properties of bloch waves and wannier functions, Phys. Rev. 115, 809 (1959), doi:10.1103/PhysRev.115.809.

[43] J. D. Cloizeaux, Energy bands and projection operators in a crystal: Analytic and asymptotic properties, Phys. Rev. 135, A685 (1964), doi:10.1103/PhysRev.135.A685.

[44] J. D. Cloizeaux, Analytical properties of $n$-dimensional energy bands and wannier functions, Phys. Rev. 135, A698 (1964), doi:10.1103/PhysRev.135.A698.

[45] J. Zak, Continuity chords of bands in solids: The diamond structure, Phys. Rev. B 25, 1344 (1982), doi:10.1103/PhysRevB.25.1344.

[46] N. F. Henry, K. Lonsdale et al., International tables for X-ray crystallography, Kynoch Press (1952).

[47] N. Marzari, A. A. Mostofi, J. R. Yates, I. Souza and D. Vanderbilt, Maximally localized wannier functions: Theory and applications, Rev. Mod. Phys. 84, 1419 (2012), doi:10.1103/RevModPhys.84.1419.

[48] J. D. Cloizeaux, Orthogonal orbitals and generalized wannier functions, Phys. Rev. 129, 554 (1963), doi 10.1103/PhysRev.129.554. 